TRANSACTIONS OF THE

AMERICAN MATHEMATICAL SOCIETY

Volume 234, Number 2, 1977

\title{
ON THE DEGREE OF CONVERGENCE OF PIECEWISE POLYNOMIAL APPROXIMATION ON OPTIMAL MESHES
}

BY

\author{
H. G. BURCHARD $\left({ }^{1}\right)$
}

\begin{abstract}
The degree of convergence of best approximation by piecewise polynomial and spline functions of fixed degree is analyzed via certain $F$-spaces $\mathrm{N}^{p, n}$ (introduced for this purpose in [2]). We obtain two o-results and use pairs of inequalities of Bernstein- and Jackson-type to prove several direct and converse theorems. For $f$ in $\mathrm{N}^{p, n}$ we define a derivative $D^{n, 0} f$ in $\mathbf{L}^{0}, \sigma=\left(n+p^{-1}\right)^{-1}$, which agrees with $D^{n} f$ for smooth $f$, and prove several properties of $D^{n, \sigma}$.
\end{abstract}

1. Introduction and summary of results. In this paper we prove direct and converse theorems for piecewise polynomial and spline approximation, of fixed degree, on optimal meshes in the $L^{p}$-norm, $1<p<\infty$. There exists an intimate relationship between this problem and certain $F$-spaces $\mathbf{N}_{0}^{p, n}$. These were first introduced, in this connection, by Burchard and Hale [2], who established direct theorems of the $O\left(k^{-n}\right)$-kind. Here, $k$ is the order of the mesh, and $n-1$ the degree of the polynomial segments. In the present paper, we further analyze the spaces $\mathrm{N}_{0}^{p, n}$. We obtain two direct " $O$ " theorems $\left(o\left(k^{-n}\right)\right.$ and $o\left(k^{-n+1}\right)$ resp.), as well as other direct and converse theorems, some of which give necessary and sufficient conditions for the degree of convergence $O\left(k^{-\theta}\right)$ with $0<\theta<n$ (the suboptimal case) or $n-1<\theta$ $(n \geq 2)$. For $\theta=n=1$ (the optimal case) direct and converse theorems do not yet match up, except for $p=\infty$, a problem solved by J. P. Kahane.

We mention that the $o\left(k^{-n+1}\right)$-result amounts to a significant weakening of the hypothesis in a theorem of Freud and Popov, which in turn is an improved extension of a theorem of Korneičuk [9]. Our $o\left(k^{-n}\right)$-theorem shows, roughly speaking, if $D^{n-1} f \in \mathbf{B V}$ and $D^{n-1} f$ is a singular function, then $f$ can be approximated more rapidly by piecewise solutions $s$ of $D^{n} s=0$ than $f$ with $D^{n-1} f \in \mathrm{AC}, D^{n} f \neq 0$ (for $\sigma<1, \sigma=\left(n+p^{-1}\right)^{-1}$ ) in $\mathbf{L}^{p}$ on a bounded interval. The converse, with accompanying direct, theorems depend on pairs of inequalities of the Bernstein- and Jackson-types. Our results are

Received by the editors October 1, 1974 and, in revised form, June 8, 1976.

AMS (MOS) subject classifications (1970). Primary 41A15, 41A25; Secondary 26A30, 26A24, 26A45, 46E10.

(') Work supported in part by the National Science Foundation under Grant NSF GP-43031.

- American Mathematical Society 1977 
analogous to the general approximation theorems proved by Butzer and Scherer [4] for the case of linear approximation in $B$-spaces, $c f$. their paper for the history of these methods. We obtain characterizations of Stečkin-type and in terms of the Peetre $K$-functional, the latter result being analogous to the classical Bernstein theorem. However, a Zamansky-type characterization seems to fail, due to the nonlinearity of the approximating classes. Finally, we prove a thoerem on asymptotically optimal knot distributions.

Taking together the results of [2] and of the present paper the significance of the spaces $\mathbf{N}_{0}^{p, n}$ for our problem appears to be established. It remains to learn more about the local properties of the functions in these spaces. We can define a derivative $D^{n, o} f$ for $f$ in $N_{0}^{p, n}(\S 5)$. This agrees with $D^{n} f$ if $D^{n-1} f \in$ $\mathbf{A C}$, and in general is an element of $\mathbf{L}^{\sigma}, \sigma \leqslant 1$. Only for $\sigma=1(p=\infty, n=1)$ is the relationship clear: $D^{1,1} f=D f$ and $\mathbf{N}_{0}^{\infty, 1}=\mathbf{A C}$. In this case also $\mathbf{N}_{0}^{\infty, 1} \subsetneq$ $\mathbf{N}^{\infty, 1}=\mathbf{C} \cap \mathbf{B V}$. This case was solved by J. P. Kahane [10]. We conjecture that $\mathbf{N}_{0}^{p, n} \subsetneq \mathbf{N}^{p, n}$ in general. However (see below for the definition of $\left.E_{p, n}(f, k)\right)$, by [2]

$$
f \in \mathbf{N}^{p, n+1} \Rightarrow \sup _{k} k^{n+1} E_{p, n+1}(f, k)<\infty,
$$

while for $\theta>0$ we show in this paper

$$
\sup k^{n+\theta} E_{p, n+1}(f, k)<\infty \Rightarrow f \in \mathbf{N}_{0}^{p, n} \text {. }
$$

In particular, then, $\mathbf{N}^{p, n+1} \subset \mathbf{N}_{0}^{p, n}$, so there are no "large" gaps. Actually our result (\$4) is slightly sharper:

$$
\sum_{k} k^{-1}\left[k^{n} E_{p, n+1}(f, k)\right]^{o}<\infty \Rightarrow f \in \mathbf{N}_{0}^{p, n},
$$

while (\$2)

$$
f \in \mathbf{N}_{0}^{p, n} \Rightarrow \lim _{k} k^{n} E_{p, n+1}(f, k)=0 .
$$

We prove that this persists for approximation by splines with simple knots. This in conjunction with our result that $D^{n-1} f \in \mathbf{B V}$ implies $f \in \mathbf{N}_{0}^{p, n}$ for $\sigma<1(\$ 3)$ gives our improvement of the Korneičuk-Freud-Popov theorem.

Next we list the principal notations and definitions as well as those results of [2] that are needed in this paper. For further details, $c f$. [2], which also contains more about the history of the problem.

Notation. By "interval" we (usually) mean a bounded open interval. Let $(a, b)$ be an interval. A mesh $u$ on $(a, b)$ is of the form $u=\left(u_{0}, \ldots, u_{k}\right)$, $a=u_{0} \leqslant \cdots<u_{k}=b$. If the inequalities are strict, $u$ is a partition.

It is convenient to also consider a mesh as a collection of intervals. Thus, $I \in u$ will always mean that $I$ is one of the intervals $\left(u_{i-1}, u_{i}\right), i=1, \ldots, k$. We write $\# u=k$ for the order, $\lambda(u)=\max _{i}\left(u_{i}-u_{i-1}\right)$ for the mesh-size of $u$. Let $n$ be a positive integer. $P^{n}(u)$ (resp. $S^{n}(u)$ ) is the collection of (real) 
piecewise polynomials (resp. splines) of degree (at most) $n-1$ on the mesh $u$. More precisely, $s \in P^{n}(u)$ iff $D^{n}\left(\left.s\right|_{I}\right)=0$ when $I \in u$, while $s \in S^{n}(u)$ iff $s \in P^{n}(u)$ and $D^{n-m-1} s$ is continuous near $u_{i}$, if $u_{i}$ is repeated exactly $m$ times in $u$. For a given interval $(a, b)$ let $\pi_{k}$ (resp. $\pi_{k}^{1}$ ) denote the collection of all meshes (resp. partitions) of order $k$ on $(a, b)$. Let $P_{k}^{n}=\cup_{u \in \pi_{k}} P^{n}(u)$, $S_{k}^{n}=\cup_{u \in \pi_{k}} S^{n}(u)$ and $S_{k}^{n, 1}=\cup_{u \in \pi_{k}^{l}} S^{n}(u)$. The members of $S_{k}^{n, 1}$ are splines with simple knots. Observe that

$$
S_{k}^{n, 1} \subset S_{k}^{n} \subset P_{k}^{n} \subset S_{n(k-1)+1}^{n} \subset S_{n k}^{n} \text {. }
$$

(If $s \in P^{n}(u)$ is usually does not matter which values are assigned to $s\left(u_{i}\right)$. But it should be clear what is meant by " $s$ is continuous at the knots" etc.)

Henceforth, fix an interval $(a, b)$. If there is a need to vary $(a, b)$ we shall say so. By $\mathbf{L}^{p}$, or $\mathbf{L}^{p}(a, b)$ if necessary, denote the usual (real) Lebesgue spaces on the interval $(a, b)$ for $0<p<\infty$. It will be convenient to write $\mathbf{X}^{p}$, or $\mathbf{X}^{p}(a, b)$, for $\mathbf{L}^{p}$ when $1 \leqslant p<\infty$, and to let $\mathbf{X}^{\infty}=\mathbf{C}[a, b]$. If $f \in \mathbf{L}^{p}, u$ a mesh on $(a, b)$ and $k$ a positive integer let $E_{p, n}(f, u)$ (resp. $E_{p, n}(f, k)$, $E_{p, n}^{1}(f, k)$ ) denote the $L^{p}$-distance of $f$ from $P^{n}(u)$ (resp. $\left.P_{k}^{n}, S_{k}^{n, 1}\right)$. In particular, $E_{p, n}(f, I)$ is simply the $\mathbf{L}^{p}(I)$-distance of $f$ from the polynomials of degree $n-1$. For $0<\tau<\infty$ and $u$ ranging over all meshes on $(a, b)$ let

$$
B_{p, n, \tau}(f, u)=\sum_{I \in u} E_{p, n}(f, I)^{\tau}, \quad B_{p, n, \tau}(f)=\limsup _{\lambda(u) \rightarrow 0} B_{p, n, \tau}(f, u) .
$$

In the last equation it clearly suffices to consider partitions. Let $\sigma=\sigma(p, n)$ $=\left(n+p^{-1}\right)^{-1}$. We abbreviate $B_{p, n}(f, u)=B_{p, n, \sigma}(f, u), B_{p, n}(f)=B_{p, n, \sigma}(f)$. Also let

$$
N_{p, n}(f)=\sup _{u} B_{p, n}(f, u),
$$

with $u$ ranging over all partitions on $(a, b)$. If necessary we designate the interval: $\|\cdot\|_{p,(a, b)}, N_{p, n}(\cdot)_{(a, b)}$, etc.

1.1 Definition [2]. Let $\mathbf{N}^{p, n}$ denote the collection of elements $f$ of $\mathbf{X}^{p}$ such that $N_{p, n}(f)<\infty$. We equip $\mathrm{N}^{p, n}$ with the norm

$$
|f|_{p, n}=\|f\|_{p}^{\sigma}+N_{p, n}(f), \quad \sigma=\sigma(p, n) .
$$

So equipped $\mathrm{N}^{p, n}$ is an $F$-space.

For basic facts regarding $F$-spaces see [8, pp. $51 \mathrm{ff}]$. One verifies without difficulty that $\mathrm{N}^{p, n}$ is a linear subspace of $\mathrm{X}^{p}, \rho(f, g)=|f-g|_{p, n}$ defines an invariant metric on $\mathbf{N}^{p, n}$ under which $\mathrm{N}^{p, n}$ is a complete metric topological vector space. In fact for $\tau<1$, in particular for $\tau=\sigma$, all the functionals $B_{p, n, \tau}(\cdot, u), B_{p, n, \tau}(\cdot), N_{p, n}(\cdot)$ satisfy the triangle inequality. They are also (positively) homogeneous of degree $\tau$ (resp. $\sigma$ ).

1.2 REMARK. The topology on $\mathrm{N}^{p, n}$ is stronger than the relative topology inherited from $X^{p}$.

Given an open interval $I$ (bounded or not) denote by $\mathbf{L}^{1, \text { loc }}$ (resp. AC, BV, 
BV $\left.^{\text {loc }}, \mathbf{W}^{n, 1}, \mathbf{W}^{n, 1,10 c}\right)$ the collection of real Borel functions on $I$ which are locally (i.e. on each compact subinterval) integrable (resp. absolutely continuous, of bounded variation, locally in BV, possess an absolutely continuous $(n-1)$ st derivative, are locally in $\left.\mathrm{W}^{n, 1}\right)$. We use freely the symbol $D^{n} f$ to denote the $n$th distribution derivative of $f$; thus, $f \in \mathbf{W}^{n, 1}$ iff $D^{n} f \in \mathbf{L}^{1}$; $f \in \mathbf{B V}$ iff $D f$ is a bounded Radon measure; etc. Functions which agree a.e. are usually identified.

It is shown in [2] that $\mathbf{W}^{n, 1} \subset \mathbf{N}^{p, n}$. Accordingly, we make the following definition.

1.3 Definition [2]. Let $\mathbf{N}_{0}^{p, n}$ denote the closure of $\mathbf{W}^{n, 1}$ in $\mathbf{N}^{p, n}$. Thus, $\mathbf{N}_{0}^{p, n}$ is an $F$-space under the norm $|\cdot|_{p, n}$.

We do not know if $\mathbf{N}_{0}^{p, n}$ is a proper subspace of $\mathbf{N}^{p, n}$ (except when $\sigma=1$, i.e., $p=\infty, n=1$, see below). By $3.3 \mathrm{~N}_{0}^{p, n}$ is not locally convex for $\sigma<1$. The following summarizes the main results of [2].

1.4 THEOREM [2]. Let $\sigma=\sigma(p, n)$. If $f \in \mathrm{N}^{p, n}$ then for $k=1,2 \ldots$

$$
k^{n} E_{p, n}(f, k) \leqslant N_{p, n}(f)^{1 / \sigma} .
$$

If $f \in \mathbf{N}_{0}^{p, n}$, then $B_{p, n}(f)=\lim _{\lambda(u) \rightarrow 0} B_{p, n}(f, u)$ and

$$
\lim _{k \rightarrow \infty} k^{n} E_{p, n}(f, k)=B_{p, n}(f)^{1 / 0} \text {. }
$$

If either $f \in \mathbf{W}^{n, 1}$ or $f \in \mathbf{X}^{p}$ with $f \in \mathbf{W}^{n, 1,10 c}$ and $\left|D^{n} f\right|$ has a locally integrable majorant in $\mathbf{L}^{\sigma}$ which is monotone near $a$ and near $b$ then $f \in \mathbf{N}_{0}^{p, n}$ and

$$
B_{p, n}(f)=c_{p, n}^{o}\left\|D^{n} f\right\|_{\sigma}^{o}, \quad c_{p, n}=E_{p, n}\left(\frac{x^{n}}{n !},(0,1)\right) .
$$

For $f$ to be in $\mathbf{N}^{p, n}$ it is sufficient that $D^{n-1} f \in \mathbf{B V} \cap \mathbf{X}^{p}$.

It is shown below (\$3), if $\sigma<1$ and $D^{n-1} f \in \mathbf{B V}$ then $f \in \mathbf{N}_{0}^{p, n}$. For $\sigma=1$, however, $\mathbf{N}_{0}^{\infty, 1}=\mathbf{W}^{1,1} \subset \mathbf{N}^{\infty, 1}=\mathbf{X}^{\infty} \cap \mathbf{B V}$. We shall frequently need the following basic lemma, which is essentially Lemma 2.6 of [2]. We may omit the proof.

1.5 Lemma. For every $f$ in $\mathrm{N}_{0}^{p, n}$ and positive $\varepsilon$ there is a positive $\delta$ such that for all partitions $u$ on $(a, b)$ with $\lambda(u)<\delta$ there is $s$ in $S^{n+1}(u)$ with $|f-s|_{p, n}$ $<\varepsilon$.

We conclude this section by giving a sharper form to some remarks in [2] on the relationship between the asymptotic behavior of $E_{p, n}(f, k)$ as $k \rightarrow \infty$ and that of corresponding quantities for spline approximation, of which the most interesting one is $E_{p, n}^{1}(f, k)$. The result, apparently part of the folklore, is that these agree, essentially. The method of proof is that of "pulling apart knots", based on a result of de Boor [5], of which we need the following slight extension. 
1.6 TheOREM. For $x, t \in \mathbf{R}$ let $g(x, t)=(x-t)_{+}^{n-1}$. For a mesh $w=$ $\left(w_{0}, \ldots, w_{n}\right)$ with $w_{0}<w_{n}$ on $\mathbf{R}$ we define the normalized $B$-spline

$$
\nu_{n}(w ; t)=g\left(w_{1}, \ldots, w_{n} ; t\right)-g\left(w_{0}, \ldots, w_{n-1} ; t\right)
$$

in terms of divided differences (well-defined via the familiar integral formula). If $w_{0}<w_{n-1}$ and $w_{1}<w_{n}$, then $v_{n}$ is a jointly continuous function of its $n+2$ arguments in a neighborhood of $w$ [5]. On the set of those $w$ for which $w_{0}<w_{n}$ the map $w \mapsto \nu_{n}(w ; \cdot)$ is still a continuous map into $\mathbf{L}^{p}(a, b), 1<p<\infty$.

Proof. For the first part of the theorem, see [5]. For the second part it will suffice to consider the map $v \mapsto \phi(v ; \cdot)$, where

$$
\phi(v ; t)=g\left(v_{1}, \ldots, v_{n} ; t\right), \quad v_{1} \leqslant v_{2} \leqslant \cdots<v_{n},
$$

and to show $\lim _{v \rightarrow v^{0}}\left\|\phi(v ; \cdot)-\phi\left(v^{0} ; \cdot\right)\right\|_{p,(a, b)}=0$ in case $v_{1}^{0}=v_{2}^{0}=\cdots=$ $v_{n}^{0}$. Using the integral representation of $\phi$ one finds for any mesh $v$ that $\phi(v ; t)$ is $(n-1)$ ! times $(n-1)$-dimensional Lebesgue measure of the set

$$
\begin{array}{r}
\left\{\left(x_{1}, \ldots, x_{n-1}\right): v_{1}+x_{1}\left(v_{2}-v_{1}\right)+\cdots+x_{n-1}\left(v_{n}-v_{n-1}\right)>t\right. \\
\left.\quad \text { and } 0 \leqslant x_{1} \leqslant \cdots \leqslant x_{n} \leqslant 1\right\},
\end{array}
$$

(omitting the trivial case $n=1$ ). This implies first that $\phi(v ; t)$ is uniformly bounded, and secondly, if $t^{-}<v_{1}^{0}<t^{+}$, then one can find a positive $\delta$ so that $\phi(v ; t)=\phi\left(v^{0} ; t\right)$ for $\left|v_{i}-v_{1}^{0}\right|<\delta(i=1, \ldots, n)$ and $t\left\langle t^{-}\right.$or $t>t^{+}$. From these facts the assertion follows immediately.

1.7 Corollary. Suppose $s \in P_{k}^{n}$, for $p=\infty$ assume in addition that $s \in$ $\mathbf{C}[a, b]=\mathbf{X}^{\infty}$. Then $s$ is in the $\mathbf{X}^{p}$-closure of $S_{k n}^{n, 1}\left(\right.$ resp. $S_{k(n-1)}^{n, 1}$ for $\left.p=\infty\right)$.

Proof. Suppose $s \in P^{n}(u), \# u=k, 1 \leqslant p<\infty$ (the proof for $p=\infty$ is similar). Construct a mesh $v=\left(v_{1}, \ldots, v_{K}\right)$, such that each distinct interior knot of $u$ occurs $n$ times in $v$. In addition, let $v$ have $n$ knots to the left of $a$ and $n$ knots to the right of $b$. Let $\nu_{n, i}(t)=v_{n}\left(v_{i}, v_{i+1}, \ldots, v_{i+n} ; t\right), i=$ $0, \ldots, K-n$. Then it is known that $s$ has a unique representation $s=$ $\sum_{i=0}^{K-n} \alpha_{i} \nu_{n, i}[13]$. Select a sequence of partitions $w^{m}=\left(w_{0}^{m}, \ldots, w_{K}^{m}\right)$ such that $v_{i}=\lim _{m \rightarrow \infty} w_{i}^{m}, i=0, \ldots, K$, and let

$$
s^{m}=\sum_{i=0}^{K-n} \alpha_{i} \nu_{n}\left(w_{i}^{m}, \ldots, w_{i+n}^{m} ; \cdot\right) .
$$

Then (the restriction to $(a, b)$ of) $s^{m}$ belongs to $S_{k n}^{n, 1}$, and by $1.6 \lim _{m \rightarrow \infty} \| s-$ $s^{m} \|_{p,(a, b)}=0$. (For $p=\infty$ interior knots of $u$ need be repeated only $n-1$ times in $v$ ).

1.8 Lemma. Suppose $f \in \mathbf{X}^{\infty}, s_{0} \in P_{k}^{n}, n \geqslant 2$. Then one can find $s_{1}$ in $P_{k}^{n} \cap \mathrm{X}^{\infty}$, such that $\left\|s_{1}-f\right\|_{\infty} \leqslant 2\left\|s_{0}-f\right\|_{\infty}$.

Proof. If $s_{0} \in P^{n}(u), u$ a partition with $\# u=k$, determine the unique $s_{2}$ 
in $P^{2}(u)$ such that, if $s_{1}=s_{0}+s_{2}$, then $s_{1}\left(u_{i}\right)=\frac{1}{2}\left[s_{0}\left(u_{i}-\right)+s_{0}\left(u_{i}+\right)\right]$ for $i=1, \ldots, k-1$ (interior knots) while $s_{1}(a)=s_{0}(a), s_{1}(b)=s_{0}(b)$. Then $s_{1} \in P_{k}^{n} \cap \mathbf{X}^{\infty}$ and

$$
\begin{aligned}
\left\|s_{2}\right\|_{\infty} & =\max _{0<i<k}\left|s_{2}\left(u_{i} \pm\right)\right| \\
& =\max _{0<i<k} \frac{1}{2}\left|s_{0}\left(u_{i}+\right)-s_{0}\left(u_{i}-\right)\right| \leqslant\left\|s_{0}-f\right\|_{\infty} .
\end{aligned}
$$

Therefore, $\left\|s_{1}-f\right\|_{\infty} \leqslant 2\left\|s_{0}-f\right\|_{\infty}$.

1.9 CoROLlaRY. For $1<p<\infty$ and for $p=\infty, n=1$

$$
E_{p, n}^{1}(f, n k) \leqslant E_{p, n}(f, k)<E_{p, n}^{1}(f, k) .
$$

For $p=\infty$ and $n \geqslant 2$

$$
\frac{1}{2} E_{\infty, n}^{1}(f,(n-1) k) \leqslant E_{\infty, n}(f, k) \leqslant E_{\infty, n}^{1}(f, k) .
$$

Hence, e.g., if $\theta>0$ then $(1 \leqslant p<\infty)$

$$
\begin{aligned}
\limsup _{k \rightarrow \infty} k^{\theta} E_{p, n}(f, k) & <\limsup _{k \rightarrow \infty} k^{\theta} E_{p, n}^{1}(f, k) \\
& <n^{\theta} \limsup _{k \rightarrow \infty} k^{\theta} E_{p, n}(f, k) .
\end{aligned}
$$

Similarly for "lim inf", or $p=\infty$. Thus by 1.4, with $\theta=n$ if $f \in \mathbf{N}_{0}^{p, n}$ then all limit points of $\left(k^{n} E_{p, n}^{1}(f, k)\right)_{k}$ lie in the interval $\left[B_{p, n}(f)^{1 / \sigma}, n^{n} B_{p, n}(f)^{1 / \sigma}\right]$.

For the proof of (1.1) note $\lim _{k \rightarrow \infty} k^{\theta} /[k / n]^{\theta}=n^{\theta}$ and

$$
E_{p, n}^{1}(f, k)<E_{p, n}^{1}\left(f, n\left[\frac{k}{n}\right]\right)<E_{p, n}\left(f,\left[\frac{k}{n}\right]\right) .
$$

Also, $[k / n]$ ranges through all positive integers, if $k$ does.

2. In this section we show that $E_{p, n+1}(f, k)=\sigma\left(k^{-n}\right)$ if $f \in \mathbf{N}_{0}^{p, n}$. By 1.9 the same result follows for $E_{p, n+1}^{1}(f, k)$. Freud and Popov [9] showed this under the much stronger hypothesis that $f \in \mathrm{W}^{n, 1}$ with $D^{n-1} f$ satisfying an $\mathrm{X}^{p_{\text {- }}}$ Zygmund condition. More on this in $\$ 3$.

2.1 ReMark. We are interested in properties of $B_{p, n, r}(f)$. As a function of the interval $(a, b), B_{p, n, \tau}(f)_{(a, b)}$ is additive in this sense: If $\alpha<\beta<\gamma$ and $f \in X^{p}(\alpha, \gamma)$ then

$$
B_{p, n, \tau}(f)_{(\alpha, \gamma)}=B_{p, n, \tau}(f)_{(\alpha, \beta)}+B_{p, n, \tau}(f)_{(\beta, \gamma)}
$$

Let us again consider a fixed interval $(a, b)$ and let $1<p<\infty$. Trivially, $B_{p, n, p}(f)=0$ for $f$ in $\mathbf{X}^{p}$. Also, for $1<p<\infty, B_{p, n, \tau}(f)$ is a nonincreasing function of $\tau$. This follows from [2, Lemma 2.9]:

$$
\lim _{\lambda(I) \rightarrow 0} E_{p, n}(f, I)=0 \text { if } f \in \mathbf{X}^{p} .
$$

Hence, if we define 


$$
\tau_{p, n}(f)=\inf \left\{\tau: B_{p, n, \tau}(f)=0\right\},
$$

then $0<\tau_{p, n}(f)<p$ and $B_{p, n, \tau}(f)=0$ for $\tau>\tau_{p, n}(f)$. Furthermore if $0 \leqslant \tau$ $<\rho<\infty$ then

$$
B_{p, n, \rho}(f, u)<\max _{I \in u} E_{p, n}(f, I)^{\rho-\tau} B_{p, n, \tau}(f, u) .
$$

If $B_{p, n, \tau}(f)<\infty$, then, using (2.1), it follows that $B_{p, n, p}(f)=0$. We have shown

$$
B_{p, n, \tau}(f)= \begin{cases}0 & \text { if } \infty>\tau>\tau_{p, n}(f), \\ \infty & \text { if } 0 \leqslant \tau<\tau_{p, n}(f) .\end{cases}
$$

This makes it an interesting problem to seek to determine $\tau_{p, n}(f)$. If $f \in \mathbf{N}^{p, n}$ then obviously $\tau_{p, n}(f) \leqslant \sigma(p, n) \leqslant 1$. One might conjecture that this holds for all $f$ in $\mathbf{X}^{p}$. If $f \in P_{k}^{n} \cap \mathbf{X}^{p}$ then $\tau_{p, n}(f)=0$ :

2.2 Lemma. If $f \in P_{k}^{n} \cap \mathbf{X}^{p}, 0<\tau<\infty$, then $B_{p, n, \tau}(f)=0$.

Proof. If $I$ is an interval, $u$ a mesh we abbreviate

$$
I \mid u \text { iff } \exists J \in u, I \subset J,
$$

otherwise $I \nmid u$. Now if $f \in P^{n}(u), \# u=k$ and if $w$ is any mesh on $(a, b)$ with $\lambda(w)<\delta$ then

$$
\begin{aligned}
B_{p, n, \tau}(f, w) & =\sum_{I \in w, I \nmid u} E_{p, n}(f, I)^{\tau} \\
& <k \sup _{I \subset(a, b), \lambda(I)<\delta} E_{p, n}(f, I)^{\tau} .
\end{aligned}
$$

The lemma follows now from (2.1).

The next result has several interesting consequences, $c f .2 .9,5.6$. 0 .

2.3 Proposition. Let $\sigma=\sigma(p, n)$ and suppose $f \in \mathbf{N}_{0}^{p, n}$. Then $B_{p, n+1, \sigma}(f)=$

Proof. Since $\sigma \leqslant 1$, the functional $B_{p, n+1, \sigma}(\cdot)$ is a seminorm ( $\left.\$ 1\right)$, which is trivially majorized by $B_{p, n}(\cdot)=B_{p, n, o}(\cdot)$. By 1.5 we can find a sequence $\left(s^{m}\right)_{m}$ where $s^{m} \in S_{m}^{n+1,1}$ such that $N_{p, n}\left(f-s^{m}\right) \rightarrow 0(m \rightarrow \infty)$. By $2.2 B_{p, n+1, \sigma}\left(s^{m}\right)$ $=0($ all $m)$. Thus for $m=1,2, \ldots$,

$$
\begin{aligned}
B_{p, n+1, \sigma}(f) & =\left|B_{p, n+1, \sigma}(f)-B_{p, n+1, \sigma}\left(s^{m}\right)\right| \\
& <B_{p, n+1, \sigma}\left(f-s^{m}\right) \leqslant B_{p, n}\left(f-s^{m}\right)<N_{p, n}\left(f-s^{m}\right) .
\end{aligned}
$$

Now let $m \rightarrow \infty$.

Of course, $\tau_{p, n+1}(f) \leqslant \tau_{p, n}(f) \leqslant \sigma(p, n)$ for $f$ in $\mathbf{N}^{p, n}$. Recall that trivially $\tau_{p, n+1}(f)<\sigma(p, n+1)<\sigma(p, n)$ for $f$ in $\mathrm{N}^{p, n+1}$ (shown to be contained in $\mathbf{N}_{0}^{p, n}$ in $\left.\$ 4\right)$.

Next, we see how $B_{p, n, \tau}(f)$ relates to the asymptotic behavior of $E_{p, n}(f, k)$. 
2.4 Definition [2]. If $u$ is any mesh on $(a, b)$, or a subinterval, let

$$
\mu_{p, n}(u, f)=\max _{I \in u} E_{p, n}(f, I) \text {. }
$$

If no confusion can arise we let $\mu(u)=\mu_{p, n}(u, f)$. We say $u$ is $E_{p, n}$-balanced for $f$ in $\mathbf{X}^{p}$ iff $E_{p, n}(f, I)=\mu(u)$ for all $I \in u$.

Given $p$ and $n$, it was shown in [2] that for each $f$ in $\mathbf{X}^{p}$ and each positive integer $k$ there is an $E_{p, n}$-balanced mesh $u^{k}$ with $\# u^{k}=k$ and that $\lim _{k \rightarrow \infty}$ $\mu\left(u^{k}\right)=0$. This is used frequently in the sequel.

2.5 Lemma. Suppose $f \in \mathbf{X}^{p}, n$ a positive integer, $0<\tau<p$. If $u$ is a mesh on $(a, b)$ then

$$
B_{p, n, \tau}(f, u)^{1 / \tau}<k^{\theta} E_{p, n}(f, u), \quad \theta=\frac{1}{\tau}-\frac{1}{p}, \quad k=\# u,
$$

with equality iff $u$ is $E_{p, n}$-balanced for $f$.

The proof is an application of Hölder's inequality. The special case $\tau=$ $\sigma(p, n), \theta=n$, is in [2].

2.6 TheOREM. Let $f \in X^{p}$. Then for $p, n, \tau, \theta$ as in 2.5

$$
\limsup _{k \rightarrow \infty} k^{\theta} E_{p, n}(f, k) \leqslant B_{p, n, \tau}(f)^{1 / \tau} .
$$

The proof would be an immediate consequence of 2.5 if it was true that $\lambda\left(u^{k}\right) \rightarrow 0(k \rightarrow \infty)$ for a sequence $\left(u^{k}\right)_{k}$ of meshes $E_{p, n}$-balanced for $f$. This holds for "most" $f$ in $\mathbf{X}^{p}$ [2, Lemma 2.11]. For general $f$ in $\mathbf{X}^{p}$ we need the following result, again required below in later sections, which is a stronger, and more general, form of [2, Theorem 2.12].

2.7 LEMMA. If $f \in \mathbf{X}^{p}$ and $\varepsilon>0$ one can find $\delta>0$ such that for all meshes $u$ on $(a, b)$ with $\mu_{p, n}(u, f)<\delta$ there exists a mesh $v$ on $(a, b)$ such that $\lambda(v)<\varepsilon$ and

$$
\left|B_{p, n, \tau}(f, u)-B_{p, n, \tau}(f, v)\right|<\varepsilon .
$$

Proof. Abbreviate again $\mu(\cdot)=\mu_{p, n}(\cdot, f)$. The proof is a refinement of the proof of [2, Theorem 2.12] and we may be brief. If $i$ is a "trivial" interval, i.e. $E_{p, n}(f, I)=0$, then $I$ is contained in a maximal (open) trivial interval. These can be arranged in a sequence $\left(V_{i}\right)_{i}, V_{i}=\left(\alpha_{i}, \beta_{i}\right)$, and $\Sigma_{i} \lambda\left(V_{i}\right)<\infty$. Let $l_{m}=\sum_{i>m} \lambda\left(V_{i}\right), m=1,2, \ldots$ Then $\left(l_{m}\right)_{m}$ is a null sequence; we do not rule out that $l_{m}=0$ for some $m$. For any mesh $u$ on $(a, b)$ let $u^{m}$ be the mesh obtained by adjoining to $u$ extra knots $\alpha_{1}, \ldots, \alpha_{m}, \beta_{1}, \ldots, \beta_{m}$, and define

$$
\lambda_{m}(u)=\max \left\{\lambda(I): I \in u^{m}, I \cap \bigcup_{i=1}^{m} V_{i}=\varnothing\right\} .
$$

One then shows 


$$
\begin{aligned}
\left|B_{p, n, \tau}(f, u)-B_{p, n, \tau}\left(f, u^{m}\right)\right| & \leqslant 2 m \mu(u)^{\tau}, \\
\limsup _{\mu(u) \rightarrow 0} \lambda_{m}(u) & \leqslant l_{m} .
\end{aligned}
$$

The proof of (2.3) is by induction over $m$, while (2.4) follows from (2.1) by a compactness argument. Let $\varepsilon>0$. First choose $m$ such that $l_{m}<\varepsilon$. By (2.4) select $\delta_{1}>0$ such that $\mu(u)<\delta_{1}$ implies $\lambda_{m}(u)<\varepsilon$. Finally choose $\delta$ such that

$$
0<\delta<\min \left\{\delta_{1},(\varepsilon / 2 m)^{1 / \tau}\right\} .
$$

Let $u$ be a mesh on $(a, b)$ with $\mu(u)<\delta$. Then $\lambda_{m}(u)<\varepsilon$. Construct a mesh $v$ from $u^{m}$ by filling in extra knots in the trivial intervals $V_{i}, i=1, \ldots, m$, say, equidistantly. This can ge done so that $\lambda(v)<\varepsilon$. Moreóver, $B_{p, n, \tau}\left(f, u^{m}\right)=$ $B_{p, n, \tau}(f, v)$. It follows now from (2.3) that $\left|B_{p, n, \tau}(f, u)-B_{p, n, \tau}(f, v)\right|<\varepsilon$.

2.8 Corollary. Let $f \in \mathbf{X}^{p}$. Then

$$
\begin{gathered}
\limsup _{\mu(u) \rightarrow 0} B_{p, n, \tau}(f, u)=B_{p, n, \tau}(f), \\
\lim _{\mu(u) \rightarrow 0} B_{p, n, \tau}(f, u)=\liminf _{\lambda(u) \rightarrow 0} B_{p, n, \tau}(f, u) .
\end{gathered}
$$

In particular $B_{p, n}(f)=\lim _{\mu(u) \rightarrow 0} B_{p, n}(f, u)$ for $f \in \mathbf{N}_{0}^{p, n}$.

Proof. Immediate from 2.7, (2.1) and for the last part, 1.4.

Proof of 2.6. Select a sequence $\left(u^{k}\right)_{k}$ of $E_{p, n}$-balanced partitions, \# $u^{k}=$ $k$, for $f$ (cf. the remark after 2.4). By $2.5 B_{p, n, \tau}\left(f, u^{k}\right)=k^{\theta} E_{p, n}\left(f, u^{k}\right) \geqslant$ $k^{\theta} E_{p, n}(f, k)$. Since $\mu\left(u^{k}\right) \rightarrow 0(k \rightarrow \infty), 2.6$ follows from 2.8 .

2.9 THEOREM. If $f \in \mathbf{N}_{0}^{p, n}$, then

$$
\lim _{k \rightarrow \infty} k^{n} E_{p, n+1}^{1}(f, k)=0 .
$$

Proof. If $f \in \mathrm{N}_{0}^{p, n}$ then $B_{p, n+1, \sigma}(f)=0$ by $2.3, \sigma=\sigma(p, n)$. By 2.6 $\lim _{k \rightarrow \infty} k^{\theta} E_{p, n+1}(f, k)=0, \theta=1 / \sigma-1 / p=n$. The theorem follows from 1.9.

Korneičuk's theorem asserts (2.5) for $f \in \operatorname{Lip}_{1}, p=\infty, n=1$. Freud and Popov proved (2.5) for general $p, n, f \in \mathrm{W}^{n, 1}$ with $D^{n-1} f$ satisfying an $\mathrm{X}^{p}$-Zygmund condition [9]. By $1.3 \mathrm{~W}^{n, 1} \subset \mathrm{N}_{0}^{p, n}$ (" $=$ " for $p=\infty, n=1$ ) hence the Zygmund condition can be removed. For $\sigma<1$ it is clear from 1.4 that $\mathbf{N}_{0}^{p, n}$ is much larger than $\mathbf{W}^{n, 1}$. In the next section this becomes more evident, cf. 3.4f.

We remark that a different and slightly more constructive proof of 2.9 can be given by first approximating $f$ in the metric of $\mathbf{N}^{p, n}$ by suitable $s$ in $S_{l}^{n+1,1}$, $l=[(k+1) / 2]$, and then finding an $E_{p, n}$-balanced approximation $\tilde{s}$ in $P_{l}^{n}$ for $f-s$ (in the $X^{p}$-metric). After that one "pulls apart" the knots of $s+\tilde{s}$, considered as element of $S_{k(n+1)}^{n+1}$. 
3. The class of functions shown to belong to $\mathbf{N}_{0}^{p, n}$ in 3.1 is already known to be included in $\mathbf{N}^{p, n}$ by 1.4 .

3.1 ThEOREM. Suppose $\sigma(p, n)<1$ and $f \in \mathrm{W}^{n-1,1}$ with $D^{n-1} f$ in $\mathrm{BV}$ on the interval $(a, b)$. Then $f \in \mathbf{N}_{b}^{p, n}$. If in addition $D^{n-1} f$ is a singular function in the sense of Lebesgue, i.e. $D^{n} f=0$ a.e., then $B_{p, n}(f)=0$ and $\lim _{k \rightarrow \infty} k^{n} E_{p, n}(f, k)$ $=0$.

For the proof we need the following result. This has to do with "relative completion", $c f$. Butzer and Nessel [3] for this concept and for similar results. Our statement makes explicit reference to approximation via convolution with an approximate identity since this is convenient for the application to be made. We may omit the proof which is along standard lines. $V(f)_{I}$ denotes the total variation of $f$ on the interval $I$.

3.2 Proposition. Let $\phi$ be a function in $\mathbf{C}^{\infty}(\mathbf{R})$ such that $\phi>0, \int_{\mathbf{R}} \phi(t) d t=$ $1, \operatorname{supp}(\phi) \subset[-1,1]$, and let $\phi_{k}(t)=k \phi(k t)$ for positive integer $k$. Assume $f \in \mathbf{W}^{n-1,1, \text { loc }}(\mathbf{R})$ with $D^{n-1} f \in \mathbf{B V}(\mathbf{R})$. Let $f_{k}=f * \phi_{k}$. If $1<p<\infty$ and $\sigma<1$ (for $\sigma=1$ add the requirement $f \in C(\mathbf{R})$ ), then $f_{k} \in \mathbf{C}^{\infty}(\mathbf{R}), \lim _{k \rightarrow \infty} \| f$ $-f_{k} \|_{p,(a, b)}=0$ and $\left\|D^{n} f_{k}\right\|_{1,(a, b)}<V\left(D^{n-1} f\right)_{\mathbf{R}}$.

Proof of 3.1. Fix $p, n$ with $\sigma=\sigma(p, n)<1$. Assume $f \in \mathrm{W}^{n-1,1}$ and $D^{n-1} f \in \mathbf{B V}$ on $(a, b)$. Then $f \in \mathrm{N}^{p, n}$ by 1.4 (if $p=\infty$, then $n>1$ and $f \in \mathbf{C}[a, b])$. By the Lebesgue decomposition theorem we can write $f=f_{a}+$ $f_{s}$ where $f_{a} \in \mathbf{W}^{n, 1}$, i.e. $D^{n} f_{a} \in \mathbf{L}^{1}$ while $D^{n-1} f_{s}$ is a singular function in the sense of Lebesgue, i.e. the $n$th distribution derivative $D^{n} f_{s}$ is a singular bounded Radon measure on $(a, b)$. Since $f_{a} \in \mathbf{N}_{0}^{p, n}$ by definition, we may assume $f_{s}=f, f_{a}=0$. Let $\mu=D^{n} f$ and suppose $\eta>0$. Since $\mu$ is a measure singular with respect to Lebesgue measure $\mathcal{L}$ we can find a positive integer $m$ and disjoint intervals $I_{1}, \ldots, I_{m}$ contained in $(a, b)$ such that for $J=\bigcup_{i=1}^{m} I_{i}$ one has

$$
\mathcal{L}(J)<\eta \text { and }|\mu|((a, b) \backslash J)<\eta,
$$

$|\mu|$ being the total variation measure of $\mu$. Define the Radon measure $\mu_{0}$ on $\mathbf{R}$ by

$$
\mu_{0}(E)=\mu(E \cap J)
$$

for Borel sets $E \subset \mathbf{R}$. Define a function $f_{0}$ by

$$
f_{0}(x)=\sum_{j=0}^{n-1} D^{j} f(a)(x-a)^{j} / j !+d_{n} \int_{(a, x)}(x-t)^{n-1} \mu_{0}(d t)
$$

$$
\text { if } x \in \mathbf{R} \text {. }
$$

Here $D^{n-1} f(a)=D^{n-1} f(a+), d_{n}=1 /(n-1) !$ and the integral vanishes for $x<a$. Then $D^{n-1} f_{0} \in \mathbf{B V}(\mathbf{R})$ and $D^{n} f_{0}=\mu_{0}$. Notice that (3.1) still holds with 
the subscript "0" if $a<x<b$. From now on we write $\|\mu\|_{V,(a, b)}$ for the total variation of the measure $\mu$. Thus, since $\mu=D^{n} f$,

$$
\|\mu\|_{V,(a, b)}=V\left(D^{n-1} f\right)_{(a, b)} .
$$

On account of

$$
\left|\left(\mu-\mu_{0}\right) E\right|=|\mu(E \backslash J)|<|\mu|(E \backslash J)
$$

for all Borel sets $E$ on $(a, b)$ we find

$$
\left\|\mu-\mu_{0}\right\|_{V,(a, b)}=\left|\mu-\mu_{0}\right|((a, b))<|\mu|((a, b) \backslash J)<\eta \text {. }
$$

This implies by [2, Lemma 2.1$]$

$$
\left\|f-f_{0}\right\|_{p,(a, b)}<M \eta, \quad N_{p, n}\left(f-f_{0}\right)_{(a, b)}<M \eta^{\sigma}
$$

for some constant $M$. It suffices, therefore, to obtain a close approximation to $f_{0}$ in $\mathbf{N}_{0}^{p, n}$. With $\phi_{k}$ as in 3.2 let $f_{k}=f_{0} * \phi_{k}, k=1,2 \ldots$ By $3.2 f_{k} \in \mathbf{C}^{\infty}(\mathbf{R})$ and

$$
\begin{gathered}
\lim _{k \rightarrow \infty}\left\|f_{0}-f_{k}\right\|_{p,(a, b)}=0, \\
\left\|D^{n} f_{k}\right\|_{1,(a, b)}<\left\|\mu_{0}\right\|_{V, \mathbf{R}} \leqslant\|\mu\|_{V,(a, b) .}
\end{gathered}
$$

Next, since $\mu_{0}$ vanishes on $\mathbf{R} \backslash J$, we see from (3.1) that $f_{0} l_{I}$ is a polynomial of degree at most $n-1$ if $I$ is an interval included in some component of $\mathbf{R} \backslash J$. Similarly, if we let $J_{k}=J+(-1 / k, 1 / k)$ and if $I$ is an interval included in some component of $\mathbf{R} \backslash J_{k}$, then

$$
\left.D^{n} f_{k}\right|_{I}=\left.\left[\left(D^{n} f_{0}\right) * \phi_{k}\right]\right|_{I}=\left.\left(\mu_{0} * \phi_{k}\right)\right|_{I}=0,
$$

since $\operatorname{supp}\left(\phi_{K}\right) \subset[-1 / k, 1 / k]$. Hence $\left.f_{k}\right|_{I}$ is a polynomial of degree at most $n-1$. It follows that for an interval $I$

$$
E_{p, n}\left(f_{0}-f_{k}, I\right)=0 \text { if } I \cap J_{k}=\varnothing .
$$

Now we can estimate $N_{p, n}\left(f_{0}-f_{k}\right)_{(a, b)}$. Let us choose $\delta$ so that $0<\delta$ $<\eta /(4 m)$. If $w$ is any partition on $(a, b)$ let

$$
\begin{aligned}
& w^{0}=\left\{I \in w: \lambda(I)<\delta \text { and } I \cap J_{k} \neq \varnothing\right\}, \\
& w^{1}=\{I \in w: \lambda(I)>\delta\} .
\end{aligned}
$$

We shall abuse notation and treat $w^{0}, w^{1}$ as meshes. Let $l=\# w^{1}$. Clearly $l<(b-a) / \delta$. Because of (3.4) we have

$$
B_{p, n}\left(f_{0}-f_{k}, w\right)=B_{p, n}\left(f_{0}-f_{k}, w^{0}\right)+B_{p, n}\left(f_{0}-f_{k}, w^{1}\right) .
$$

Estimating as in 2.5 , i.e. by Hölder's inequality,

$$
\begin{aligned}
B_{p, n}\left(f_{0}-f_{k}, w^{1}\right) & <l^{n \sigma} E_{p, n}\left(f_{0}-f_{k}, w^{1}\right)^{\circ} \\
& <(b-a)^{n \sigma} \delta^{-n \sigma}\left\|f_{0}-f_{k}\right\|_{p,(a, b)}^{\sigma} .
\end{aligned}
$$

Also, by a different application of Hölder's inequality, as in [2, Lemma 2.1], 
and since $\mathcal{L}\left(J_{k}\right)<\eta+2 m / k$,

$$
\begin{aligned}
B_{p, r}\left(f_{0}-f_{k}, w^{0}\right) & \leqslant d_{n}^{\sigma}\left(\sum_{I \in w^{0}} \lambda(I)\right)^{1-\sigma}\left(\sum_{I \in w^{0}}\left\|D^{n} f_{0}-D^{n} f_{k}\right\|_{V, I}\right)^{\sigma} \\
& \leqslant d_{n}^{\sigma}(\eta+2 m / k+2 m \delta)^{1-\sigma}\left\|\mu_{0}-D^{n} f_{k}\right\|_{V,(a, b)}^{o} \\
& \leqslant d_{n}^{\sigma}\left(\frac{3}{2} \eta+2 m / k\right)^{1-\sigma} 2^{\sigma}\|\mu\|_{V,(a, b)}^{o}
\end{aligned}
$$

(the last inequality by the choice of $\delta$ and by (3.3)). Combining these estimates and taking the sup over all $w$ we get

$$
\begin{aligned}
N_{p, n}\left(f_{0}-f_{k}\right)_{(a, b)} \leqslant & d_{n}^{\sigma}\left(\frac{3}{2} \eta+2 m / k\right)^{1-\sigma} 2^{\sigma}\|\mu\|_{V,(a, b)}^{\sigma} \\
& +(b-a)^{n \sigma} \delta^{-n \sigma}\left\|f_{0}-f_{k}\right\|_{p,(a, b) .}^{\sigma}
\end{aligned}
$$

From this and (3.3) we see that we can choose $k$ such that for some constant $M_{1}$ (depending on $f$ ):

$$
\left\|f_{0}-f_{k}\right\|_{p,(a, b)}<\eta \text { and } N_{p, n}\left(f_{0}-f_{k}\right)_{(a, b)}<M_{1} \eta^{1-\sigma} \text {. }
$$

Combining this with (3.2) and recalling $0<\sigma<1$ we obtain an estimate $\left|f-f_{k}\right|_{p, n,(a, b)}<\psi(\eta)$ where $\lim _{\eta \rightarrow 0+} \psi(\eta)=0$. This shows $f \in \mathbf{N}_{0}^{p, n}$. For the proof of the remainder of the theorem, if again $D_{a}^{n-1} f$ is a singular function and $\eta, m, J, f_{0}, \delta$ are as above and $w$ is a partition of $(a, b)$ with $\lambda(w)<\delta$, let $w^{0}=\{I \in w: I \cap J \neq \varnothing\}$. Then, estimating as in (3.5) and using (3.2),

$$
\begin{aligned}
B_{p, n}(f, w) & <B_{p, n}\left(f-f_{0}, w\right)+B_{p, n}\left(f_{0}, w\right) \\
& <N_{p, n}\left(f-f_{0}\right)+d_{n}^{\sigma}\left(\sum_{I \in w^{0}} \lambda(I)\right)^{1-\sigma}\left(\sum_{I \in w^{0}}\left\|D^{n} f_{0}\right\|_{V, I}\right)^{\sigma} \\
& <M \eta^{\sigma}+d_{n}^{\sigma}(\eta+2 m \delta)^{1-\sigma}\left\|\mu_{0}\right\|_{V}^{\sigma} \\
& <M \eta^{\sigma}+d_{n}^{\sigma}\left(\frac{3}{2} \eta\right)^{1-\sigma}\|\mu\|_{V}^{\sigma}=\psi_{1}(\eta) .
\end{aligned}
$$

Again, $\lim _{\eta \rightarrow 0+} \psi_{1}(\eta)=0$, thus $B_{p, n}(f)=0$. By 1.4 this implies

$$
\lim _{k \rightarrow \infty} k^{n} E_{p, n}(f, k)=0 .
$$

An examination of the preceding proof will show that if $f$ satisfies the hypothesis of 3.1 and $f_{1}$ suitably extends $f$ to $\mathbf{R}$ (e.g. as in (3.1) with the subscript " 0 " dropped) then

$$
\lim _{k \rightarrow \infty}\left|f_{1}-f_{1} * \phi_{k}\right|_{p, n,(a, b)}=0 .
$$

We conjecture that no such result can hold for all $f$ in $\mathbf{N}_{0}^{p, n}$. In this connection the following result is of interest. This also depends on Proposition 5.1 below. 
For simplicity we consider $\mathbf{N}_{0}^{p, n}(T), T$ the circle identified with $[-\pi, \pi)$. Let $\phi \in C^{\infty}(T), \phi \geqslant 0, \int_{T} \phi d t=1, \operatorname{supp}(\phi) \subset[-0.1,0.1]$.

3.3 Corollary. If $f \in N_{0}^{p, n}(T)$, the map $t \rightarrow f_{t}=f(\cdot-t)$ is continuous from $T$ to $\mathbf{N}_{0}^{p, n}(T)$, and

$$
f * \phi=\int_{T} f_{t} \phi(t) d t
$$

exists as an $\mathrm{X}^{p}(T)$-valued Riemann integral. Yet, for some $f$ in $\mathrm{N}_{0}^{p, n}(T), f * \phi$ does not exist as an $\mathbf{N}_{0}^{p, n}(T)$-valued Riemann integral, no sequence of Riemann sums for $f * \phi$ with mesh-size tending to zero converges in $N_{0}^{p, n}(T)$ and $F=\left\{f_{t}\right.$ : $t \in T\}$, though compact, does not have a relatively compact convex hull in $\mathbf{N}_{0}^{p, n}(T)$. It follows that $\mathbf{N}_{0}^{p, n}(T)$ is not locally convex. (In all this $\sigma(p, n)<1$.)

Proof. Let $g$ be the standard Cantor function on $[0,1], f_{1}(t)=g\left(\frac{1}{2}\right.$ $+\frac{1}{2} \cos t$ ), and define recursively

$$
f_{n+1}(t)=\int_{0}^{t} f_{n}(x) d x-\frac{t}{2 \pi} \int_{-\pi}^{\pi} f_{n}(x) d x,
$$

$n=1,2, \ldots,-\pi \leqslant t \leqslant \pi$. Then $D^{n-1} f_{n} \in \mathbf{C} \cap \mathbf{B V}$, but $D^{n-1} f_{n}$ is singular, $D^{n} f_{n}$ is a positive singular measure on $(-\pi, 0)$, negative on $(0, \pi)$. For a Riemann-sum $R(u)$ for $f * \phi, f=f_{n}$,

$$
R(u)=\sum_{I \in u} f_{t_{I}} \phi\left(t_{I}\right) \lambda(I), \quad t_{I} \in I,
$$

over the partition $u$ of $T$, we have $D^{n, \sigma} R(u)=0$ by 3.1 and 5.1. If a sequence $\left(u^{k}\right)_{k}$ of partitions of $T$ is given with $\lambda\left(u^{k}\right) \rightarrow 0$ as $k \rightarrow \infty$, then if $\left(R\left(u^{k}\right)\right)_{k}$ is a convergent sequence in $N_{0}^{p, n}(T)$, then by 1.2 it is so in $\mathrm{X}^{p}(T)$, with identical limit, which must be $f * \phi$ (as ordinary Lebesgue integral). This gives

$$
\begin{aligned}
\left(D^{n} f\right) * \phi & =D^{n}(f * \phi)=D^{n, \sigma}(f * \phi) \\
& =\lim _{k \rightarrow \infty}\left(L^{\circ}\right)^{n, \sigma} R\left(u^{k}\right)=0,
\end{aligned}
$$

which is impossible since the distribution derivative $\left(D^{n} f\right) * \phi$ is a positive measure (in fact $\left.C^{\infty}\right)$ on $(-\pi+0.1,-0.1)$. The special Riemann-sums $R(u)$ with $t_{I}$ so chosen that $\int_{I} \phi(t) d t=\phi\left(t_{I}\right) \lambda(I)$ are in the convex hull $\operatorname{cv}(F)$ of $F$, and therefore $\operatorname{cv}(F)$ cannot be relatively compact. It follows that $\mathrm{N}_{0}^{p, n}(T)$ is not locally convex [8].

It is remarkable that the preceding proof does not depend on the failure of $\mathbf{L}^{\sigma}$ to be locally convex. This fact does imply, however, that $D^{n, \sigma}$ does not have a continuous linear right inverse, i.e., $\operatorname{ker} D^{n, \sigma}=\left\{f \in \mathrm{N}_{0}^{p, n}: B_{p, n}(f)=\right.$ $0\}$ is not complemented in $\mathbf{N}_{0}^{p, n}$. It follows from the Baire category theorem that the map $f \mapsto f * \phi$ is continuous from $L^{1}(T)$ to $\mathbf{N}_{0}^{p, n}(T)$, hence from $\mathbf{N}_{0}^{p, n}(T)$ to $\mathbf{N}_{0}^{p, n}(T)$. 
3.4 Corollary. Suppose $\sigma<1$ and $f \in \mathrm{W}^{n-1,1}$ with $D^{n-1} f \in \mathrm{BV}$. Then $\lim _{k \rightarrow \infty} k^{n} E_{p, n+1}^{1}(f, k)=0$. If moreover $D^{n-1} f$ is a singular function then $\lim _{k \rightarrow \infty} k^{n} E_{p, n}^{1}(f, k)=0$.

This follows immediately from 1.9, 2.9 and the preceding result. Corollary 3.4 lends further emphasis to the fact that 2.9 is a considerable improvement of the Korneičuk-Freud-Popov theorem, $c f$. remarks following 2.9. The result in 3.4 that $E_{p, n}(f, k)=o\left(k^{-n}\right)$ if $D^{n-1} f$ is a singular function, i.e. $D^{n} f=0$ a.e. is what one would hope for since the approximators in $S_{k}^{n, 1}$ are precisely those functions which have this property in the stronger form " $D^{n-1} f \in \mathrm{BV}, D^{n} f=0$ piecewise". By contrast, if $f \in \mathrm{W}^{n, 1}$, i.e. $D^{n-1} f \in \mathbf{A C}$, then by $1.4,1.9$

$$
\begin{aligned}
B_{p, n}(f)^{1 / \sigma} & =c_{p, n}\left\|D^{n} f\right\|_{\sigma} \text { and }\left(\forall f \in \mathbf{N}_{0}^{p, n}\right) \\
B_{p, n}(f)^{1 / \sigma} & \leqslant \liminf _{k \rightarrow \infty} k^{n} E_{p, n}^{1}(f, k) \\
& \leqslant \limsup _{k \rightarrow \infty} k^{n} E_{p, n}^{1}(f, k)<2 n^{n} B_{p, n}(f)^{1 / \sigma} .
\end{aligned}
$$

3.5 Corollary. Suppose $\sigma<1, f \in \mathrm{W}^{n-1,1}$ and $D^{n-1} f \in$ BV. Then $f=f_{a}$ $+f_{s}$, with $D^{n-1} f_{a} \in \mathbf{A C}$ and $D^{n-1} f_{s}$ singular, and

$$
B_{p, n}(f)=B_{p, n}\left(f_{a}\right)=c_{p, n}^{\sigma}\left\|D^{n} f_{a}\right\|_{\sigma}^{\sigma}=\lim _{k \rightarrow \infty} k^{n} E_{p, n}(f, k) .
$$

This is an immediate consequence of 3.1 and 1.4. The following is a companion of 3.5, which sharpens part of [2, Theorem 2.8].

3.6 Corollary. Suppose $\sigma<1, f \in \mathrm{W}^{n-1,1,10 c} \cap \mathbf{X}^{p}$ and $D^{n-1} f \in \mathbf{B V}^{\text {loc }}$. Then $f=f_{a}+f_{s}$ with $D^{n-1} f_{a} \in \mathrm{AC}^{\mathrm{loc}}$ and $D^{n-1} f_{s}$ singular,

$$
\liminf _{\lambda(u) \rightarrow 0} B_{p, n}(f, u)=c_{p, n}^{0}\left\|D^{n} f_{a}\right\|_{o}^{o}
$$

and $\lim \inf _{k \rightarrow \infty} k^{n} E_{p, n}(f, k) \geqslant c_{p, n}\left\|D^{n} f_{a}\right\|_{o}^{\sigma}$ (the right-hand expression may be $\infty)$

We omit the proof. The result corresponding to (3.6) in [2] had only " $>$ ", but in fact "=" can be shown.

3.7 The case $\sigma=1$. In this case, $p=\infty, n=1$, and one can show that for all $f$ in $\mathbf{X}^{\infty}=C[a, b]$

$$
\begin{aligned}
\frac{1}{2} V(f) & =N_{\infty, 1}(f)=B_{\infty, 1}(f) \\
& =\lim _{\lambda(u) \rightarrow 0} B_{\infty, 1}(f, u)=\lim _{\mu(u) \rightarrow 0} B_{\infty, 1}(f, u)
\end{aligned}
$$

with $\mu(u)=\mu_{\infty, 1}(u, f)$, whether or not any of the quantities in (3.7) are finite. It follows that

$$
\lim _{k \rightarrow \infty} k E_{\infty, 1}(f, k)=\frac{1}{2} V(f) \quad\left(\text { all } f \text { in } \mathbf{X}^{\infty}\right) .
$$


Note that $E_{\infty, 1}^{1}(f, k)=E_{\infty, 1}(f, k)$. The result (3.8) is due to J. P. Kahane [10]. We remark that for $\sigma<1$ the analog of the second equality in (3.7) is patently false by 3.1. The third and fourth equalities in (3.7) have been shown for $\sigma<1$ only if $f \in \mathbf{N}_{0}^{p, n}$. We omit the proofs.

A comment is due regarding another result of J. P. Kahane in [10]. There, the quantity

$$
V^{\psi}(f)=\sup _{u} \sum_{i=1}^{\# u} \psi\left(\left|f\left(u_{i}\right)-f\left(u_{i-1}\right)\right|\right)
$$

is introduced, with the sup taken over all partitions $u$ on $(a, b), \psi$ being a continuous strictly increasing subadditive function on $[0, \infty)$ with $\psi(0)=0$. Kahane proves a result analogous to (3.8) for $V^{\psi}(f)$. However, this is not essentially more general than (3.8). Namely, either

$$
\limsup _{x \rightarrow 0+} \psi(x) / x=\infty
$$

and then $V^{\psi}(f)<\infty$ implies $f$ is constant, or (3.9) is false; but then $V^{\psi}(f)<\infty$ iff $V(f)<\infty$, for $f$ in $\mathrm{X}^{\infty}$.

Finally, it is clear from (3.7) that $\mathbf{N}^{\infty, 1}=\mathbf{B V} \cap \mathbf{X}^{\infty}$ and $\mathbf{N}_{0}^{\infty, 1}=\mathbf{A C}$. If this case is typical we conjecture that $\mathbf{N}^{p, n}$ is the relative completion of $\mathbf{N}_{0}^{p, n}$ in $\mathbf{X}^{p}$, cf. 3.2 and the remark preceding it (however, for $\sigma<1, N_{0}^{p, n}$ is not a $B$-space). It is easy to see that the relative completion of $\mathbf{N}_{0}^{p, n}$ in $\mathbf{X}^{p}$ is included in $\mathbf{N}^{p, n}$.

4. In this section we prove degree of approximation theorems of the type established by Butzer and Scherer [4] in the context of linear approximation in Banach spaces, and analogous to classical results of Jackson, Bernstein and Stečkin. A Zamansky-type result, as contained in [4], appears to fail due to the nonlinearity of the approximating classes $P_{k}^{n}$. The proofs depend on pairs of inequalities of the Bernstein- and Jackson-types, as in [4]. The method of "pulling apart knots," $c f .1 .7$ and 1.8, and a Bernstein-type inequality also give the result that for $f$ to be an element of $\mathbf{N}_{0}^{p, n}$ it suffices that $f \in \mathbf{N}_{0}^{p, n}(I)$ for each interval $I$ of a partition on $(a, b)$, and that $f \in \mathbf{X}^{\infty}$ in case $p=\infty$.

The next three lemmas establish inequalities of the Bernstein-type. Here $\mathrm{N}^{p, n}$ acts as the "smooth" space.

4.1 Lemma. If $s \in P_{k}^{n}, \sigma=\sigma(p, n)$ then $N_{p, n}(s)^{1 / \sigma}<k^{n}\|s\|_{p}$.

Proof. Suppose $s \in P^{n}(u), u$ a mesh on $(a, b)$ with $\# u=k$ and let $w$ be any partition on $(a, b)$. Using the notation of (2.2) and using Hölder's inequality as in the proof of 2.5 , for $1 \leqslant p<\infty$ 


$$
\begin{aligned}
B_{p, n}(s, w) & =\sum_{I \in w, I \nmid u} E_{p, n}(s, I)^{\sigma} \\
& <k^{n \sigma}\left(\sum_{I \in w, I \nmid u} E_{p, n}(s, I)^{p}\right)^{\sigma / p} \\
& <k^{n \sigma} E_{p, n}(s, w)^{\sigma}<k^{n \sigma}\|s\|_{p}^{\sigma} .
\end{aligned}
$$

Similarly for $p=\infty$.

If $s \in P_{k}^{n} \cap \mathbf{X}^{p}$ this shows $s \in \mathbf{N}^{p, n}$ (by 4.5 actually $s \in \mathbf{N}_{0}^{p, n}$ ).

4.2 LEMMA. If $s \in P_{k}^{n+1}$ then

$$
c_{p, n}\left\|D^{n} s\right\|_{0} \leqslant k^{n}\|s\| p .
$$

For $c_{p, n}$, see $1.4 D^{n} s$ is defined only in the interior of mesh-intervals.

Proof. Suppose $s \in P^{n+1}(u), u$ a partition on $(a, b)$, \# $u=k$. If $I$ is an interval, $I \mid u$, then

$$
c_{p, n} \lambda(I)^{1 / \sigma}\left|D^{n} s\left(x_{I}\right)\right|=E_{p, n}(s, I), \quad x_{I} \in I
$$

(this is a trivial consequence of the definition of $\left.c_{p, n}\right)$. Hence, also using 2.5,

$$
\begin{aligned}
c_{p, n}\left\|D^{n} s\right\|_{\sigma}^{\sigma} & =\sum_{I \in u} c_{p, n}^{\sigma} \lambda(I)\left|D^{n} s\left(x_{I}\right)\right|^{\sigma} \\
& =\sum_{I \in u} E_{p, n}(s, I)^{\sigma}=B_{p, n}(s, u) \\
& <k^{n \sigma} E_{p, n}(s, u) \leqslant k^{n \sigma}\|s\|_{p}^{\sigma} .
\end{aligned}
$$

4.3 LEMMA. If $s \in P_{k}^{n+1}$ then $N_{p, n}(s)^{1 / \sigma} \leqslant 2^{1 / \sigma} k^{n}\|s\|_{p}$.

Proof. Again, if $s \in P^{n+1}(u)$, \#u=k, and $w$ is any mesh, then by (4.1), and estimating as in the proof of 4.1

$$
\begin{aligned}
B_{p, n}(x, w) & =\sum_{I \in w, I \mid u} c_{p, n}^{\sigma} \lambda(I)\left|D^{n} s\left(x_{I}\right)\right|^{\sigma}+\sum_{I \in w, I \nmid u} E_{p, n}(s, I)^{\circ} \\
& \leqslant c_{p, n}^{\sigma}\left\|D^{n} s\right\|_{\sigma}^{\sigma}+k^{n \sigma} E_{p, n}(s, w)^{\sigma} .
\end{aligned}
$$

By 4.2 this implies the lemma.

4.4 RemarK. As is easy to see, if $f \in \mathbf{X}^{p}$, and $\left.f\right|_{I} \in \mathrm{N}^{p, n}(I)$ for all $I$ in $u, u$ a partition on $(a, b)$, then $f \in \mathbf{N}^{p, n}$. The corresponding property for $\mathbf{N}_{0}^{p, n}$ lies deeper.

4.5 THEOREM.If $f$ belongs piecewise to $\mathbf{N}_{0}^{p, n}$, i.e., $f \in \mathbf{X}^{p}$ and $\left.f\right|_{I} \in \mathbf{N}_{0}^{p, n}(I)$ for all $I$ in $u, u$ a partition on $(a, b)$, then $f \in \mathbf{N}_{0}^{p, n}$. In particular $P_{k}^{m} \cap \mathbf{X}^{p} \subset \mathbf{N}_{0}^{p, n}$ for positive integers $m, k$.

Proof. If $\sigma=1$, then $\mathbf{N}_{0}^{p, n}=\mathbf{A C}, c f$. 3.7. It is easy to see that the theorem holds in this case. Let $\sigma<1$. Thus $p=\infty$ implies $n>2$. Observe that it 
suffices to consider the case $\# u=2$, since $\mathbf{N}_{0}^{p, n}$ is a linear space. Hence let $u=(a, c, b)$ and $I_{1}=(a, c), I_{2}=(c, b), f \in \mathbf{X}^{p},\left.f\right|_{L_{1}} \in \mathbf{N}_{0}^{p, n}\left(I_{j}\right), j=1,2$. By 1.5 we can find sequences $\left(s_{j}^{l}\right)_{l}, j=1,2, s_{j}^{l} \in S_{l}^{n+1,1}$ such that $\lim _{l \rightarrow \infty} \mid f-$ $\left.s_{j}^{\prime}\right|_{p, n, l_{j}}=0$. Let us piece $s_{1}^{l}$ and $s_{2}^{l}$ together:

$$
s^{l}(t)=s_{j}^{l}(t) \quad \text { if } t \in I_{j}, j=1,2 .
$$

It is then clear that $s \in \mathbf{L}^{p}$ and that $\lim _{l \rightarrow \infty}\left\|s^{l}-f\right\|_{p,(a, b)}=0$. For $p=\infty, s^{l}$ need not be continuous. However, one can in this case choose $s_{j}^{l}$ such that $s_{1}^{l}(c)=s_{2}^{l}(c)$ (use 4.3 and the method of proof of 1.8), ensuring $s^{l} \in \mathbf{X}^{\infty}$. Thus, in any case, $f$ is the $\mathbf{X}^{p}$-limit of the sequence $\left(s^{l}\right)_{l}$. Furthermore, as is easily seen,

$$
N_{p, n}\left(s^{l}-f\right)_{(a, b)} \leqslant N_{p, n}\left(s_{1}^{l}-f\right)_{I_{1}}+n_{p, n}\left(s_{2}^{l}-f\right)_{I_{2}}+\left\|s^{\prime}-f\right\|_{p,(a, b)}^{0} .
$$

By hypothesis, this shows that $f$ is the $\mathbf{N}^{p, n}$-limit of the sequence $\left(s^{l}\right)_{l}$. It suffices, therefore, to show that $s^{l} \in \mathrm{N}_{0}^{p, n}, l=1,2 \ldots$ Since $s^{\prime} l_{l_{j}}=s_{j}^{l} \in$ $\mathrm{W}^{n, 1}\left(I_{j}\right)$, this reduces the proof of the theorem to the case that $f \in P_{k}^{n+1} \cap$ $\mathbf{X}^{p}$, for some $k$. Let us assume this is so. By 1.7 we can find a sequence $\left(f^{m}\right)_{m}$ in $S_{k(n+1)}^{n+1,1} \subset \mathbf{N}_{0}^{p, n}$ such that $\lim _{m \rightarrow \infty}\left\|f-f^{m}\right\|_{p}=0$. Notice that

$$
f^{m}-f^{l} \in P_{2 k(n+1)}^{n+1}, \quad m, l=1,2, \ldots
$$

Hence by 4.3

$$
N_{p, n}\left(f^{m}-f^{l}\right) \leqslant 2[2 k(n+1)]^{n o}\left\|f^{m}-f^{l}\right\|_{p}^{o} .
$$

We have shown that $\left(f^{m}\right)_{m}$ is Cauchy in $\mathbf{N}_{0}^{p, n}$. By 1.2 this implies that $f$ is the limit in $\mathbf{N}_{0}^{p, n}$ of the sequence $\left(f^{m}\right)_{m}$, thus $f \in \mathbf{N}_{0}^{p, n}$.

It is natural to expect that $\mathrm{N}_{0}^{p, n}$ should possess the property expressed in 4.5 in view of its relationship to piecewise polynomial approximation. If fact 4.5 enters in an essential way into the proof of the next result.

4.6 REMARK. Lemmas 4.1, 4.2, 4.3 have the form of Bernstein's inequality, if we consider $N_{p, n}(s)$, or $\left\|D^{n} s\right\|_{\sigma}$, as a measure of smoothness of $s$. In complete analogy with the framework described by Butzer and Scherer [4], the inequalities of 4.1 and 4.3 (not 4.2) can be paired with inequalities of Jackson's type. These follow from 1.4:

$$
\begin{gathered}
E_{p, n}(f, k) \leqslant k^{-n} N_{p, n}(f)^{1 / \sigma}, \\
E_{p, n+1}(f, k) \leqslant k^{-n} N_{p, n}(f)^{1 / \sigma},
\end{gathered}
$$

for $f$ in $\mathbf{N}^{p, n}$, the second inequality following trivially from the first. Next we obtain a characterization of Stečkin-type from inequalities 4.3 and (4.3).

Existence of best approximations in $P_{k}^{n+1}$ for $f$ in $\mathbf{X}^{p}$ is shown in [2] $(k=1$, $2, \ldots)$. Given $p$ and $n$ with $1<p<\infty$ we select $s_{k}(f)$ in $P_{k}^{n+1}\left(\subset \mathbf{X}^{p}\right)$ such that 


$$
E_{p, n+1}(f, k)=\left\|f-s_{k}(f)\right\|_{p}, \quad 1<p<\infty .
$$

For $p=\infty$ we choose $s_{k}(f)$ in $P_{k}^{n+1} \cap \mathrm{X}^{\infty}$ by 1.8 such that

$$
E_{\infty, n+1}(f, k) \leqslant\left\|f-s_{k}(f)\right\|_{\infty}<2 E_{\infty, n+1}(f, k) .
$$

In either case we observe that $s_{k}(f) \in \mathbf{N}_{0}^{p, n}$ by 4.5. For a real sequence $\left(c_{k}\right)_{k}$ we employ the notation $\left\|c_{k}\right\|_{(k) q}$ for the $l^{q}$-norm and we let for $1<q<\infty$

$$
\left\|c_{k}\right\|_{(k) \bullet q}=\left\{\sum_{k=1}^{\infty} \frac{1}{k}\left|c_{k}\right|^{q}\right\}^{1 / q},
$$

while $\left\|c_{k}\right\|_{(k), \infty}=\left\|c_{k}\right\|_{(k) \infty}$.

4.7 TheOREM. Let $\sigma=\sigma(p, n), 1 \leqslant q \leqslant \infty, \theta>n \sigma$. If $f \in \mathbf{X}^{p}$, the following are equivalent:

$$
\begin{gathered}
\left\|k^{\theta} E_{p, n+1}(f, k)^{\sigma}\right\|_{(k) \cdot q}<\infty, \\
f \in \mathbf{N}_{0}^{p, n} \text { and }\left\|k^{\theta-n o} N_{p, n}\left(f-s_{k}(f)\right)\right\|_{(k) \cdot q}<\infty, \\
f \in \mathbf{N}_{0}^{p, n} \text { and }\left\|2^{k(\theta-n o)} N_{p, n}\left(f-s_{2^{k}}(f)\right)\right\|_{(k) q}<\infty .
\end{gathered}
$$

Furthermore, (4.6) implies

$$
\sum_{k=1}^{\infty} \frac{1}{k}\left[k^{n} E_{p, n+1}(f, k)\right]^{\sigma}<\infty
$$

(i.e., (4.6) with $q=1, \theta=n \sigma$ ), and (4.9) is sufficient for $f$ to be in $\mathbf{N}_{0}^{p, n}$.

Proof. Throughout the proof we write $s_{k}=s_{k}(f)$. (4.6) $\Rightarrow(4.8)$ : Assume (4.6) holds. We first show this implies (4.9). By Hölder's inequality, if $1 / q+1 / q^{\prime}=1$, then for $c_{k}=k^{n \sigma} E_{p, n+1}(f, k)^{\sigma}$

$$
\sum_{k=1}^{\infty} \frac{1}{k} c_{k} \leqslant\left\|k^{\theta-n \sigma} c_{k}\right\|_{(k), q}\left\|k^{n \sigma-\theta}\right\|_{(k) \bullet q^{\prime}}
$$

Since $\left\|k^{n \sigma-\theta}\right\|_{(k) \bullet q^{\prime}}<\infty$, this. proves (4.9). We leave it to the reader to verify that for $1<q<\infty, \theta>0$ (and letting $M_{\alpha}$ denote, here and below, various relative constants)

$$
\left\|2^{k \theta} E_{p, n+1}\left(f, 2^{k}\right)^{\sigma}\right\|_{(k) q} \leqslant M_{0}\left\|k^{\theta} E_{p, n+1}(f, k)^{\sigma}\right\|_{(k) \bullet q^{\circ}}
$$

For $q=1, \theta=n \sigma$ this and (4.9) give

$$
\sum_{k=1}^{\infty} 2^{k n \sigma} E_{p, n+1}\left(f, 2^{k}\right)^{\sigma}<\infty .
$$

Now observe that

$$
s_{2^{k+1}}-s_{2^{k}} \in P_{3 \cdot 2^{k^{k}}}^{n+1} .
$$

Hence, by 4.3 and (4.4) or (4.5) 


$$
\begin{aligned}
N_{p, n}\left(s_{2^{k+1}}-s_{2^{k}}\right) & \leqslant 2 \cdot 3^{n \sigma} 2^{k n \sigma}\left\|s_{2^{k+1}}-s_{2^{k}}\right\|_{p}^{\sigma} \\
& \leqslant 2 \cdot 3^{n \sigma} 2^{k n \sigma}\left\{\left\|f-s_{2^{k+1}}\right\|_{p}^{\sigma}+\left\|f-s_{2^{k}}\right\|_{p}^{\sigma}\right\} \\
& \leqslant M_{1} 2^{k n \sigma} E_{p, n+1}\left(f, 2^{k}\right)^{\sigma} .
\end{aligned}
$$

It follows that for $m>k$

$$
N_{p, n}\left(s_{2^{m}}-s_{2^{k}}\right) \leqslant M_{1} \sum_{l=k}^{m-1} 2^{l n o} E_{p, n+1}\left(f, 2^{l}\right)^{\circ} .
$$

Since, of course, $\lim _{k \rightarrow \infty}\left\|f-s_{k}\right\|_{p}=0,(4.11)$ and (4.12) show that $\left(s_{2^{m}}\right)_{m}$ is a Cauchy sequence in $\mathrm{N}^{p, n}$, and in fact by 1.2 and $4.5\left(s_{2^{m}}\right)_{m}$ converges to $f$ in $\mathbf{N}_{0}^{p, n}$, thus $f \in \mathbf{N}_{0}^{p, n}$. We at once obtain from (4.12)

$$
N_{p, n}\left(f-s_{2^{k}}\right) \leqslant M_{1} \sum_{l=k}^{\infty} 2^{l n \sigma} E_{p, n+1}\left(f, 2^{l}\right)^{\sigma} .
$$

This implies, using Minkowski's inequality, for $n \sigma<\theta$,

$$
\begin{aligned}
\left\|2^{k(\theta-n \sigma)} N_{p, n}\left(f-s_{2^{k}}\right)\right\|_{(k) q} & \leqslant M_{1}\left\|\sum_{l>k} 2^{(l-k)(n \sigma-\theta)} 2^{l \theta} E_{p, n+1}\left(f, 2^{l}\right)^{\sigma}\right\|_{(k) q} \\
& \leqslant M_{1} \sum_{l>0} 2^{l(n \sigma-\theta)}\left\|2^{(k+l) \theta} E_{p, n+1}\left(f, 2^{k+l}\right)^{\sigma}\right\|_{(k) q} \\
& \leqslant \frac{M_{1}}{1-2^{n \sigma-\theta}}\left\|2^{k \theta} E_{p, n+1}\left(f, 2^{k}\right)^{\sigma}\right\|_{(k) q^{\cdot}}
\end{aligned}
$$

Now (4.6) and (4.10) imply (4.8).

(4.8) $\Rightarrow(4.7)$. Assume (4.8). Let $2^{m} \leqslant k<2^{m+1}, m>1$. Then we show

$$
N_{p, n}\left(f-s_{k}\right) \leqslant M_{4}\left\{N_{p, n}\left(f-s_{2^{m-1}}\right)+N_{p, n}\left(f-s_{2^{m+1}}\right)\right\} \text {. }
$$

By the Jackson-type inequality (4.3)

$$
E_{p, n+1}(f, 2 k-1)^{0} \leqslant E_{p, n+1}\left(f-s_{k}, k\right)^{0}<k^{-n o} N_{p, n}\left(f-s_{k}\right),
$$

and therefore

$$
E_{p, n+1}\left(f, 2^{m}\right)^{\sigma}<2^{-(m-1) n \sigma} N_{p, n}\left(f-s_{2^{m-1}}\right) .
$$

This and 4.3 imply (observing $s_{2^{m+1}}-s_{k} \in P_{2^{m+2}}^{n+1}$ ):

$$
\begin{aligned}
N_{p, n}\left(s_{2^{m+1}}-s_{k}\right) & <2\left(2^{m+2}\right)^{n \sigma}\left\|s_{2^{m+1}}-s_{k}\right\|_{p}^{\sigma} \\
& <M_{2} 2^{m n \sigma}\left\{\left\|s_{2^{m+1}}-f\right\|_{p}^{\sigma}+\left\|s_{k}-f\right\|_{p}^{\sigma}\right\} \\
& <M_{3} 2^{m n \sigma} E_{p, n+1}\left(f, 2^{m}\right)^{\sigma}<M_{4} N_{p, n}\left(f-s_{2^{m-1}}\right) .
\end{aligned}
$$

From this we obtain (4.13). Next, (4.13) gives

$$
\begin{aligned}
& k^{\theta-n o} N_{p, n}\left(f-s_{k}\right) \\
& \quad<M_{5}\left\{2^{(m-1)(\theta-n \sigma)} N_{p, n}\left(f-s_{2^{m-1}}\right)+2^{(m+1)(\theta-n \sigma)} N_{p, n}\left(f-s_{2^{m+1}}\right)\right\}
\end{aligned}
$$


for $2^{m}<k<2^{m+1}$. For $q=\infty$ it follows directly from the preceding inequality that (4.8) implies (4.7). For $1 \leqslant q<\infty$ we estimate (ignoring $k=1)$

$$
\begin{aligned}
\sum_{k=2}^{\infty} \frac{1}{k} & {\left[k^{\theta-n o} N_{p, n}\left(f-s_{k}\right)\right]^{q} } \\
& =\sum_{m=1}^{\infty} \sum_{k=2^{m}}^{2^{m+1}} \frac{1}{k}\left[k^{\theta-n o} N_{p, n}\left(f-s_{k}\right)\right]^{q} \\
& <M_{\xi}^{q} \sum_{m=1}^{\infty}\left[2^{(m-1)(\theta-n o)} N_{p, n}\left(f-s_{2^{m-1}}\right)+2^{(m+1)(\theta-n o)} N_{p, n}\left(f-s_{2^{m+1}}\right)\right]^{q} \\
& <M_{6}\left\|2^{m(\theta-n o)} N_{p, n}\left(f-s_{2^{m}}\right)\right\|_{(m) q}^{q}<\infty
\end{aligned}
$$

by Minkowski's inequality and by (4.8). This proves (4.7).

$(4.7) \Rightarrow(4.6)$. Assume (4.7). The Jackson-type inequality (4.14) and the obvious relation $E_{p, n+1}(f, 2 k) \leqslant E_{p, n+1}(f, 2 k-1)$ give

(4.15) $j^{\theta} E_{p, n+1}(f, j)^{\sigma}<2^{\theta} k^{\theta-n o} N_{p, n}\left(f-s_{k}\right), \quad j=2 k-1$ or $j=2 k$.

Using this and (4.7) we can estimate

$$
\left\|j^{\theta} E_{p, n+1}(f, j)^{\sigma}\right\|_{(j) * q} \leqslant M_{7}\left\|k^{\theta-n o} N_{p, n}\left(f-s_{k}\right)\right\|_{(k) \cdot q}
$$

This completes the proof of the theorem.

4.8. REMARK. Our proof of 4.7 differs from the corresponding treatment by Butzer and Scherer [4] in several details. The main difference is that in our case there does not appear to be an analog of inequality [4, (3.12)]. We overcome this in part by our inequality (4.13), no analog of which occurs in [4]. However, it is for this reason that we are unable to prove a Zamanskytype characterization.

4.9 REMARK. (4.14), and hence (4.15), is correct whenever $s_{k} \in P_{k}^{n+1} \cap \mathrm{X}^{p}$. This shows that (4.7) is also equivalent to (4.16):

$$
\begin{aligned}
& f \in \mathbf{N}_{0}^{p, n} \text { and }\left\|k^{\theta-n o} N_{p, n}\left(f-s_{k}\right)\right\|_{(k) * q}<\infty \\
& \text { for some sequence }\left(s_{k}\right)_{k}, s_{k} \in P_{k}^{n+1} \cap \mathbf{X}^{p} .
\end{aligned}
$$

Next, we obtain several interesting corollaries.

4.10 Corollary. Let $1<p<\infty, n$ a positive integer. Then $\mathbf{N}^{p, n+1} \subset \mathbf{N}_{0}^{p, n}$. More precisely, if $f \in \mathbf{X}^{p}$ and $0<\theta<1$ then

$$
\begin{aligned}
f \in \mathbf{N}^{p, n+1} & \Rightarrow E_{p, n+1}(f, k)=O\left(k^{-n-\theta}\right) \\
& \Rightarrow \sum_{k=1}^{\infty} \frac{1}{k}\left[k^{n} E_{p, n+1}(f, k)\right]^{\sigma}<\infty \Rightarrow f \in \mathbf{N}_{0}^{p, n} \\
& \Rightarrow E_{p, n}(f, k)=O\left(k^{-n}\right) .
\end{aligned}
$$


Proof. By 1.4 and 4.7.

4.11 Corollary. Let $p=\infty, n$ a positive integer, $f \in \mathbf{X}^{\infty}$. If (4.6) holds $(\theta>n \sigma, 1<q<\infty)$ or (4.9) holds then $f \in \mathbf{A C}$. The following are equivalent for $\theta>1$ :

$$
\begin{gathered}
\left\|k^{\theta} E_{\infty, 2}(f, k)\right\|_{(k) * q}<\infty, \\
f \in \mathbf{A C} \text { and }\left\|k^{\theta-1} V\left(f-s_{k}\right)\right\|_{(k) * q}<\infty, \\
f \in \mathrm{AC} \text { and }\left\|2^{k(\theta-1)} V\left(f-s_{2^{k}}\right)\right\|_{(k) q}<\infty .
\end{gathered}
$$

Here, $s_{k}=s_{k}(f) \in P_{k}^{2} \cap \mathbf{X}^{\infty}=S_{k}^{2,1}$, and (4.5) holds with $n=1$.

Proof. By $3.7 N_{\infty, 1}(f)=\frac{1}{2} V(f)$ and $N_{0}^{\infty, 1}=$ AC. Now apply 4.7 and 4.10.

This result can be improved as follows. It is almost trivial that for a polynomial $s$ of degree $n$

$$
V(s)_{I}=\|D s\|_{1, I}<M_{n}\|s\|_{\infty, I}, \quad M_{n}=2 n .
$$

This immediately implies on the interval $(a, b)$

$$
V(s) \leqslant M_{n} k\|s\|_{\infty}, \quad s \in P_{k}^{n+1} \cap \mathrm{X}^{\infty} .
$$

This inequality of Bernstein-type can be paired with the Jackson-type inequality

$$
E_{\infty, n+1}(f, k) \leqslant 2^{-1} k^{-1} V(f) \text { if } f \in \mathbf{X}^{\infty},
$$

which follows from 1.4 and (3.7). There is no difficulty in carrying over the method of proof of 4.7 , line by line, to the present situation, replacing the inequality pair 4.3, (4.3) by (4.20), (4.21). The result is the following theorem.

4.12 Theorem. Let $1 \leqslant q \leqslant \infty, \theta>1$. If $f \in \mathrm{X}^{\infty}$, the following are equivalent:

$$
\begin{gathered}
\left\|k^{\theta} E_{\infty, n+1}(f, k)\right\|_{(k) * q}<\infty, \\
f \in \mathbf{A C} \text { and }\left\|k^{\theta-1} V\left(f-s_{k}(f)\right)\right\|_{(k), q}<\infty, \\
f \in \mathbf{A C} \text { and }\left\|2^{k(\theta-1)} V\left(f-s_{2^{k}}(f)\right)\right\|_{(k) q}<\infty .
\end{gathered}
$$

Furthermore, (4.22) implies

$$
\sum_{k=1}^{\infty} E_{\infty, n+1}(f, k)<\infty
$$

(i.e., (4.22) with $q=1, \theta=1$ ) and (4.25) is sufficient for $f \in \mathrm{AC}$. Here, $s_{k}(f)$ is as in (4.5). In addition (4.22) holds iff

$$
\left\|k^{\theta-1} E_{1, n}(D f, k)\right\|_{(k) \bullet q}<\infty .
$$


The proof of the last part is as in 4.9, noting that $V(f)=\|D f\|_{1}$ for $f$ in AC. We point out that 4.12 reduces the case of $p=\infty$ to that of $p=1$. We can directly relate (4.26) to (4.6) for $q=\infty$ (not for $1<q<\infty$ ). We thus obtain the following result.

4.13 CoRollary. Let $n>1, \theta>n, f \in \mathrm{X}^{\infty}$. The following are equivalent:

$$
\begin{gathered}
\sup _{k} k^{\theta} E_{\infty, n+1}(f, k)<\infty, \\
f \in \mathrm{AC} \text { and } \sup _{k} k^{\theta-1} E_{1, n}(D f, k)<\infty,
\end{gathered}
$$

$f \in \mathrm{AC}, D f \in \mathbf{N}_{0}^{1, n-1}$ and $\sup _{k} k^{\theta-n} N_{1, n-1}\left(D f-s_{k}^{(1)}\right)^{n}<\infty$,

where $s_{k}^{(1)} \in P_{k}^{n},\left\|D f-s_{k}^{(1)}\right\|_{1}=E_{1, n}(D f, k)$.

$$
f \in \mathrm{N}_{0}^{\infty, n} \text { and } \sup _{k} k^{\theta-n} N_{\infty, n}\left(f-s_{k}\right)^{n}<\infty,
$$

where $s_{k}=s_{k}(f) y P_{k}^{n+1} \cap \mathrm{X}^{\infty}$ satisfying (4.5)

The proof follows from 4.7 and $4.12(q=\infty)$, noting $\sigma(\infty, n)=1 / n=$ $\sigma(1, n-1)$. We observe that

$$
N_{\infty, n}(f)<N_{1, n-1}(D f) \text { for } f \in \mathrm{AC} .
$$

Thus, the implication $(4.29) \Rightarrow(4.30)$ follows directly.

Next, we use the Peetre $K$-functional to obtain a characterization analogous to the classical Bernstein theorem.

4.14 Definition. Let $1<p<\infty$ and $n$ be a positive integer, $\sigma=\sigma(p, n)$. If $f \in \cdot \mathbf{X}^{p}, 0<t<\infty$, let

$$
K_{p, n}(t, f)=\inf \left\{\|f-g\|_{p}^{\sigma}+t N_{p, n}(g): g \in \mathbf{N}^{p, n}\right\} .
$$

This functional, if not a special case, is at least closely analogous to the $K$-functional introduced by Peetre in the context of Banach spaces, cf. [4] for references.

We state the following theorem, omitting the proof, which is based on the Bernstein-type inequality 4.1 and the Jackson-type inequality (4.2), and which does not present any novel problems. We only consider the case $q=\infty$.

4.15 Theorem. Let $0<\theta<1, f \in \mathbf{X}^{p}, 1<p<\infty$. The following are equivalent:

$$
\begin{aligned}
& \sup _{k} k^{\theta n} E_{p, n}(f, k)<\infty, \\
& \sup _{0<t<1} t^{-\theta} K_{p, n}(t, f)<\infty .
\end{aligned}
$$

We add the following comments. $K_{p, n}(t, f)$ is analogous to a higher modulus of continuity. It is not hard to see that the following are equivalent for $f$ in $\mathrm{X}^{p}$ : (i) $f$ is a polynomial of degree $n-1$ or less; (ii) $N_{p, n}(f)=0$; (iii) 
$K_{p, n}(t, f)=0$ for some $t$ in $(0, \infty)$; (iv) $\lim _{t \rightarrow 0+} t^{-1} K_{p, n}(t, f)=0$. Furthermore, if (4.32) holds with $\theta=1$, then $f \in N^{p, n}$ (and conversely) and thus (4.31) holds with $\theta=1$. The latter property implies, as in Bernstein's theorem,

$$
\sup _{0<t<2^{-n}}[t|\log t|]^{-1} K_{p, n}(t, f)<\infty,
$$

which is, of course, weaker than (4.32) with $\theta=1$.

4.16 Remark. Examples of functions in $X^{p} \backslash N^{p, n}$ are most easily constructed using (3.6), since it is shown in [2] that $f \in N^{p, n}$ iff $f \in X^{p}$ and $B_{p, n}(f)<\infty$. Thus, an example in [1] which is in $\mathbf{W}^{1,1,10 c} \cap \mathbf{L}^{1}$ but has $\|D f\|_{1 / 2}=\infty$ is in $\mathbf{L}^{1} \backslash \mathbf{N}^{1,1}$. As shown in [7], $f(t)=|\log (t)|^{-1}$, belonging to $\mathbf{X}^{\infty}\left(0, \frac{1}{2}\right)$, has $\left\|D^{2} f\right\|_{1 / 2}=\infty$ hence is in $\mathbf{X}^{\infty} \backslash \mathbf{N}^{\infty, 2}$. The latter example has a monotone and negative second derivative.

5. In this section we consider the dependence of $B_{p, n}\left(f_{(a, b)}\right.$ on the interval $(a, b)$. This quantity is of interest because of 1.4. Besides, it was shown in [2] that if $f \in \mathbf{X}^{p}$, then $f \in \mathbf{N}^{p, n}$ iff $B_{p, n}(f)<\infty$.

5.1 Proposition. Let $1 \leqslant p<\infty, n$ a positive integer. There is a linear, continuous map $D^{n, \sigma}: \mathrm{N}_{0}^{p, n} \rightarrow \mathbf{L}^{\sigma}, \sigma=\sigma(p, n)$, such that $D^{n, \sigma} f=D^{n} f$ if $f \in$ $\mathrm{W}^{n, 1}$ and

$$
B_{p, n}(f)_{I}=c_{p, n}^{\sigma}\left\|D^{n, \sigma} f\right\|_{\sigma, I}^{\sigma}
$$

for every interval $I \subset(a, b)$. These properties determine $D^{n, o}$ uniquely.

Proof. Let $f_{1}, f_{2} \in \mathbf{W}^{n, 1}$. By 1.4

$$
c_{p, n}^{\sigma}\left\|D^{n} f_{1}-D^{n} f_{2}\right\|_{\sigma}^{\sigma}=B_{p, n}\left(f_{1}-f_{2}\right)<N_{p, n}\left(f_{1}-f_{2}\right) .
$$

Being uniformly continuous from $\mathbf{W}^{n, 1}$ with $\mathrm{N}_{0}^{p, n}$-metric to $\mathbf{L}^{0}, D^{n}$ has a unique continuous linear extension, $D^{n, \sigma}: \mathbf{N}_{0}^{p, n} \rightarrow \mathbf{L}^{\sigma}$. Also, for an interval $I \subset(a, b)$, if $\left(f_{k}\right)_{k}$ is a sequence in $\mathbf{W}^{n, 1}$ converging to $f$ in $\mathbf{N}_{0}^{p, n}$,

$$
\begin{aligned}
\left|c_{p, n}^{0}\left\|D^{n, \sigma} f\right\|_{\sigma, I}^{0}-B_{p, n}\left(f_{k}\right)_{I}\right| & =c_{p, n}^{\sigma}\left|\left\|D^{n, \sigma} f\right\|_{\sigma, I}^{\sigma}-\left\|D^{n} f_{k}\right\|_{\sigma, I}^{\sigma}\right| \\
& <c_{p, n}^{\sigma}\left\|D^{n, o} f-D^{n} f_{k}\right\|_{\sigma, I}^{\sigma} \rightarrow 0 \text { as } k \rightarrow \infty,
\end{aligned}
$$

hence $B_{p, n}(f)_{I}=\lim _{k \rightarrow \infty} B_{p, n}\left(f_{k}\right)_{I}=c_{p, n}^{\sigma}\left\|D^{n, o} f\right\|_{\sigma, I}^{0}$.

5.2 Corollary. If $f \in \mathbf{N}_{0}^{p, n}$, then $B_{p, n}(f)_{I}$ is an absolutely continuous interval function.

Proof. $B_{p, n}(f)_{I}=c_{p, n}^{\sigma} \int_{l}\left|D^{n, \sigma} f(t)\right|^{\sigma} d t$, and $\left|D^{n, \sigma} f\right|^{\sigma} \in \mathbf{L}^{1}$.

Note that $n$ and $\sigma$ determine $p$, so there is no ambiguity in the notation $D^{n, \sigma}$. By 3.5 if $f \in W^{n-1,1}, D^{n-1} f \in \mathrm{BV}$, then $D^{n, \sigma} f=D^{n} f_{a}$, where $D^{n-1} f_{a}$ is the absolutely continuous part of $D^{n-1} f$. In particular, $D^{n, o} f=0$ if $D^{n-1} f$ is singular (if $\sigma<1$ ). 1.4 shows that $D^{n, o} f$ may be nonintegrable.

As was observed in the proof of 4.2, if $s \in P^{n+1}(u)$ then 


$$
c_{p, n}^{\sigma}\left\|D^{n} s\right\|_{\sigma}^{\sigma}=B_{p, n}(s, u),
$$

with $D^{n} s$ defined in the interior of the intervals of $u$. This suggests an interesting method of computing $D^{n, 0} f$, and hence $B_{p, n}(f)$. In preparation, we prove several lemmas.

5.3 Lemma. If $f \in \mathrm{N}_{0}^{p, n}$ and $u$ a partition on $(a, b)$ we denote by $s_{u}(f)$ a best approximation to $f$ in $P^{n+1}(u)$ in the $L^{p}$-metric (which is unique for $1<p<$ $\infty)$, i.e.

Then for $f$ in $\mathbf{N}_{0}^{p, n}$

$$
E_{p, n+1}(f, u)=\left\|f-s_{u}(f)\right\|_{p}
$$

$$
B_{p, n}(f)=\lim _{\lambda(u) \rightarrow 0} c_{p, n}^{\sigma}\left\|D^{n} S_{u}(f)\right\|_{\sigma}^{\sigma} .
$$

Proof. We have (5.1) and

$$
\begin{aligned}
\mid B_{p, n}(f, u) & -B_{p, n}\left(s_{u}(f), u\right) \mid \leqslant B_{p, n}\left(f-s_{u}(f), u\right) \\
= & \sum_{I \in u} E_{p, n}\left(f-s_{u}(f), I\right)^{\sigma}=\sum_{I \in u} E_{p, n+1}(f, I)^{\circ} \\
= & B_{p, n+1, \sigma}(f, u) .
\end{aligned}
$$

By $2.3, \lim _{\lambda(u) \rightarrow 0} B_{p, n+1, \sigma}(f, u)=B_{p, n+1, \sigma}(f)=0$.

This lemma is already of considerable interest, since it shows that, at least for $f \in \mathbf{N}_{0}^{p, n}, B_{p, n}(f)$ can be approximated via the "inscribed polygons of best approximation" $s_{u}(f)$ of degree $n$. However, more can be said.

5.4 Lemma. Suppose $f \in \mathbf{C}^{n}[a, b], I \subset(a, b), d_{n+1}=1 / n !, \sigma=\sigma(p, n)$. Then $E_{p, n+1}(f, I)<d_{n+1} \lambda(I)^{1 / 0} \omega\left(D^{n} f, \lambda(I)\right)$.

Proof. Let $I=(\alpha, \beta)$. Let $s(x)=\sum_{j=0}^{n} D^{j} f(\alpha)(x-\alpha)^{j} / j$ !. By Taylor's formula,

$$
|s(x)-f(x)| \leqslant d_{n+1} \lambda(I)^{n} \omega\left(D^{n} f, \lambda(I)\right), \quad x \in I .
$$

For $p=\infty$, this is the assertion. For $1<p<\infty$,

$$
\|s-f\|_{p, I} \leqslant d_{n+1} \lambda(I)^{n} \omega\left(D^{n} f, \lambda(I)\right)\left[\int_{\alpha}^{\beta} d t\right]^{1 / p},
$$

which gives the lemma.

5.5 Lemma. In the situation of 5.4, if $s_{I}$ is the (unique) best $\mathbf{L}^{p}$-approximation to $f$ in $P^{n+1}(I)$, then

$$
\left\|D^{n_{S_{I}}}-D^{n} f\right\|_{\infty, I} \leqslant M_{p, n} \omega\left(D^{n} f, \lambda(I)\right)
$$

for some constant $M_{p, n^{*}}$.

Proof. By a result of G. M. Phillips [12], cf. [2, (1.8)], if $g \in \mathbf{C}^{n}[a, b]$, then 


$$
E_{p, n}(g, I)=c_{p, n} \lambda(I)^{1 / \sigma}\left|D^{n} g(\xi)\right|,
$$

for some $\xi$ in $[\alpha, \beta], I=(\alpha, \beta)$. Thus,

$$
E_{p, n+1}(f, I)=E_{p, n}\left(f-s_{I}, I\right)=c_{p, n} \lambda(I)^{1 / q}\left|D^{n}\left(f-s_{I}\right)(\xi)\right|
$$

and hence for $t \in I$, since $D^{n} s_{I}$ is constant,

$$
\begin{aligned}
\left|D^{n} s_{I}(t)-D^{n} f(t)\right| & \leqslant\left|D^{n} s_{I}(\xi)-D^{n} f(\xi)\right|+\left|D^{n} f(\xi)-D^{n} f(t)\right| \\
& \leqslant E_{p, n+1}(f, I) /\left[c_{p, n} \lambda(I)^{1 / \sigma}\right]+\omega\left(D^{n} f, \lambda(I)\right) \\
& <M_{p, n} \omega\left(D^{n} f, \lambda(I)\right),
\end{aligned}
$$

by $5.4, M_{p, n}=\left(1+d_{n+1} / c_{p, n}\right)$.

\subsection{COROLlaRY. If $f \in \mathbf{C}^{n}[a, b]$, then}

$$
\left\|D^{n} f-D^{n} s_{u}(f)\right\|_{\infty} \leqslant M_{p, n} \omega\left(D^{n} f, \lambda(u)\right) \text {, }
$$

for all partitions $u$ on $(a, b)$. Again, $D^{n} s_{u}(f)$ is undefined at the knots of $u$.

We now obtain a much sharper statement than Lemma 5.3.

5.6 THEOREM. If $f \in \mathbf{N}_{0}^{p, n}, \sigma=\sigma(p, n)$, then

$$
\lim _{\lambda(u) \rightarrow 0}\left\|D^{n, \sigma} f-D^{n} s_{u}(f)\right\|_{\sigma}=0 .
$$

Proof. Let $f \in \mathbf{N}_{0}^{p, n}$. By [2, Lemma 2.1], if $f \in \mathbf{W}^{n, 1}$, then $N_{p, n}(f)<d_{n}^{o}(b$ $-a)^{1-\sigma}\left\|D^{n} f\right\|_{1}^{o}$. Since $\mathbf{C}[a, b]$ is dense in $\mathbf{L}^{1}$, we conclude that $\mathbf{N}_{0}^{p, n}$ is in fact the $\mathbf{N}^{p, n}$-closure of $\mathbf{C}^{n}[a, b]$. Thus, find a sequence $\left(f_{k}\right)_{k}$ in $\mathbf{C}^{n}[a, b]$ such that $\lim _{k \rightarrow \infty}\left|f-f_{k}\right|_{p, n}=0$. If $u$ is any partition on $(a, b)$, then

$$
\begin{aligned}
& \left\|D^{n, \sigma} f-D^{n} s_{u}(f)\right\|_{\sigma}^{\sigma} \\
& \quad<\left\|D^{n, \sigma} f-D^{n} f_{k}\right\|_{\sigma}^{\sigma}+\left\|D^{n}\left[s_{u}(f)-s_{u}\left(f_{k}\right)\right]\right\|_{\sigma}^{\sigma}+\left\|D^{n} f_{k}-D^{n} s_{u}\left(f_{k}\right)\right\|_{\sigma}^{\sigma} .
\end{aligned}
$$

By 5.1 and (5.1) this gives

$$
\begin{gathered}
c_{p, n}^{\sigma}\left\|D^{n, \sigma} f-D^{n} s_{u}(f)\right\|_{0}^{\sigma} \leqslant N_{p, n}\left(f-f_{k}\right)+B_{p, n}\left(s_{u}(f)-s_{u}\left(f_{k}\right), u\right) \\
+(b-a)\left\|D^{n} f_{k}-D^{n} s_{u}\left(f_{k}\right)\right\|_{\infty}^{0} .
\end{gathered}
$$

The third term on the right is bounded by

$$
(b-a) M_{p, n}^{o} \omega\left(D^{n} f_{k}, \lambda(u)\right)^{\sigma}
$$

on account of 5.5. Also, estimating as in the proof of 5.3

$$
\begin{aligned}
B_{p, n}\left(s_{u}(f)-s_{u}\left(f_{k}\right), u\right) \leqslant & B_{p, n+1, \sigma}(f, u)+B_{p, n+1,0}\left(f_{k}, u\right) \\
& +N_{p, n}\left(f-f_{k}\right) .
\end{aligned}
$$

Combining these inequalities, 


$$
\begin{aligned}
c_{p, n}^{\sigma}\left\|D^{n, \sigma} f-D^{n} s_{u}(f)\right\|_{\sigma}^{\sigma} \leqslant & 2 N_{p, n}\left(f-f_{k}\right)+(b-a) M_{p, n}^{\sigma} \omega\left(D^{n} f_{k}, \lambda(u)\right)^{\sigma} \\
& +B_{p, n+1, \sigma}(f, u)+B_{p, n+1, \sigma}\left(f_{k}, u\right) .
\end{aligned}
$$

If now $\varepsilon>0$, we can choose $k$ so large that $N_{p, n}\left(f-f_{k}\right)<\varepsilon / 8$ and a positive $\delta$ such that each of the last three terms on the right in the above inequality is $<\varepsilon / 4$ for $\lambda(u)<\delta$, since $D^{n} f_{k} \in \mathbf{C}[a, b]$ and by 2.3 . This shows

$$
c_{p, n}^{\sigma}\left\|D^{n, o} f-D^{n} s_{u}(f)\right\|_{\sigma}^{\sigma}<\varepsilon \quad \text { if } \lambda(u)<\delta .
$$

It is now very tempting to conjecture that 5.6 has a converse, namely, that convergence in $\mathbf{L}^{\sigma}$ of $D^{n} s_{u}(f)$ implies $f \in \mathbf{N}_{\delta}^{p, n}$ or at least $f \in \mathbf{N}^{p, n}$. Also, does $s_{u}(f)$ converge to $f$ in $\mathbf{N}_{0}^{p, n}$ (as $\lambda(u) \rightarrow 0$ )? We are unable to answer these questions. At any rate, 5.1 and 5.6 suggest a certain similarity between $\mathbf{N}_{0}^{p, n}$ and the Sobolev spaces $\mathrm{W}^{m, q}, q \geqslant 1$. Of course for $\sigma=1, D^{1,1}=D^{1}$ and by (3.7), $\mathbf{N}_{0}^{\infty, 1}=\mathbf{A C}=\mathbf{W}^{1,1}$.

We next prove a result on asymptotically optimal knot distributions. We cannot yet prove its usefulness for computational purposes. This, however, has been done under much more restrictive assumptions by McClure [11], Burchard [1], de Boor [6] and Dodson [7].

5.7 Theorem. (i) Suppose $f \in \mathbf{N}_{0}^{p, n}$, and $B_{p, n}(f)_{(a, b)} \neq 0$. Let $H(t)=$ $B_{p, n}(f)_{(a, t)} / B_{p, n}(f)_{(a, b)}, a \leqslant t<b . H$ is the uniform limit of functions $H_{k}$ defined as follows: For each $k=1,2, \ldots$ let $u^{k}$ be an $E_{p, n}$-balanced partition for $f$ on $(a, b), c f .2 .4 \mathrm{ff}, \# u^{k}=k, u^{k}=\left(u_{0}^{k}, \ldots, u_{k}^{k}\right)$, let $I_{j}^{k}=\left(u_{j-1}^{k}, u_{j}^{k}\right)$ and define

$$
\begin{gathered}
g_{k}(t)=\frac{E_{p, n}\left(f, I_{j}^{k}\right)^{\sigma}}{\lambda\left(I_{j}^{k}\right)}=\frac{B_{p, n}\left(f, u^{k}\right)}{k \lambda\left(I_{j}^{k}\right)} \quad \text { if } t \in I_{j}^{k}, j=1, \ldots, k, \\
H_{k}(t)=\int_{a}^{t} g_{k}(x) d x / B_{p, n}\left(f, u^{k}\right) .
\end{gathered}
$$

Each of $H, H_{k}$ is the absolutely continuous distribution function of a probability measure on $(a, b)$. The density $G$ of $H$ is given by

$$
G(t)=\left|D^{n, \sigma} f(t)\right|^{\sigma} /\left\|D^{n, \sigma} f\right\|_{\sigma,(a, b)^{\circ}}^{\sigma}
$$

Furthermore $H_{k}\left(u_{j}^{k}\right)=j / k$, the proportion of intervals of $u^{k}$ to the left of $u_{j}^{k}$.

(ii) The partitions $u^{k}$ are asymptotically optimal:

$$
\lim _{k \rightarrow \infty} k^{n} E_{p, n}(f, k)=\lim _{k \rightarrow \infty} k^{n} E_{p, n}\left(f, u^{k}\right)=B_{p, n}(f)_{(a, b)}^{1 / o} \in(0, \infty) \quad[2] \text {. }
$$

(iii) The uniform convergence $\left(H_{k}\right)_{k} \rightarrow H$ implies the weak*-convergence of the corresponding probability measures $\left(\nu_{k}\right)_{k} \rightarrow \nu$, in the weak topology of the dual of $\mathbf{C}_{0}(a, b)=\{\phi \in \mathbf{C}[a, b]: \phi(a)=\phi(0)=0\}$.

Proof. (i) Evidently $H, H_{k}$ are absolutely continuous, nondecreasing, 
vanish at $a$ and take the value 1 at $b$. Furthermore $\mu_{p, n}\left(u^{k}, f\right)$ tends to zero, $k \rightarrow \infty, c f .2 .4$, and as shown in [2]

$$
\lim _{k \rightarrow \infty} B_{p, n}\left(f, u^{k}\right)=B_{p, n}(f)_{(a, b)} \text {. }
$$

(This follows from 2.8.) By hypothesis $B_{p, n}(f)_{(a, b)}>0$. To prove uniform convergence of $\left(H_{k}\right)_{k}$ to $H$, it therefore suffices to show uniform convergence of $\left(h_{k}\right)$ to $h$, where

$$
\begin{gathered}
h(t)=B_{p, n}(f)_{(a, t)}, \\
h_{k}(t)=\int_{a}^{t} g_{k}(x) d x=\sum_{j=1}^{l-1} E_{p, n}\left(f, I_{j}^{k}\right)^{\sigma}+\frac{t-u_{l-1}^{k}}{\lambda\left(I_{l}^{k}\right)} E_{p, n}\left(f, I_{l}^{k}\right)^{\sigma}
\end{gathered}
$$$$
\text { if } t \in\left[u_{l-1}^{k}, u_{l}^{k}\right] \text {. }
$$

For $u_{l-1}^{k}<t<u_{l}^{k}$ let us introduce the partition

$$
u^{k}(t)=\left(u_{0}^{k}, \ldots, u_{l-1}^{k}, t\right)
$$

on $(a, t)$. Also let $I^{k}(t)=\left(u_{l-1}^{k}, t\right)$. Then

$$
B_{p, n}\left(f, u^{k}(t)\right)=\sum_{j=1}^{l-1} E_{p, n}\left(f, I_{j}^{k}\right)^{0}+E_{p, n}\left(f, I^{k}(t)\right)^{0} .
$$

This leads to the estimate

$$
\left|B_{p, n}\left(f, u^{k}(t)\right)-h_{k}(t)\right|<2 \mu\left(u^{k}\right)^{\sigma},
$$

$\mu(\cdot)=\mu_{p, n}(\cdot, f)$. Furthermore, again for $u_{l-1}^{k}<t<u_{l}^{k}$, let $w^{k}(t)$ be the partition

$$
\left(u_{0}^{k}, \ldots, u_{l-1}^{k}, t, w_{l+1}^{k}, \ldots, w_{m}^{k}\right)
$$

with $w_{m}^{k}=b$ and such that $v^{m}(t)=\left(t, w_{l+1}^{k}, \ldots, w_{m}^{k}\right)$ is equidistant. Here, $m=m^{k}(t)$ is chosen so large that, according to 1.4 and (2.1)

$$
\left|B_{p, n}(f)_{(t, b)}-B_{p, n}\left(f, v^{m}(t)\right)\right|<\mu\left(u^{k}\right)^{\sigma},
$$

and

$$
\mu\left(v^{m}(t)\right)<\mu\left(u^{k}\right)^{\circ}
$$

This implies, with (5.3),

$$
\begin{aligned}
\left|h(t)-h_{k}(t)\right| & <\left|h(t)-B_{p, n}\left(f, u^{k}(t)\right)\right|+2 \mu\left(u^{k}\right)^{0} \\
& \quad\left|B_{p, n}(f)_{(a, b)}-B_{p, n}\left(f, w^{k}(t)\right)\right| \\
& \quad+\left|B_{p, n}(f)_{(t, b)}-B_{p, n}\left(f, v^{m}(t)\right)\right|+2 \mu\left(u^{k}\right)^{0} \\
& <\left|B_{p, n}(f)_{(a, b)}-B_{p, n}\left(f, w^{k}(t)\right)\right|+3 \mu\left(u^{k}\right)^{0} .
\end{aligned}
$$


By (5.4), $\mu\left(w^{k}(t)\right) \leqslant \mu\left(u^{k}\right)$. Since $\mu\left(u^{k}\right)$ tends to zero, it now follows from 1.4 and 2.8 that $\lim _{k \rightarrow \infty}\left\|h-h_{k}\right\|_{\infty}=0$.

(ii) This is shown in [2]. The result follows also from 1.4, 2.5 and 2.8.

(iii) Since $\mathbf{C}_{0}(a, b)$ is separable, the probability (Radon-)measures form a metrizable compact space in the weak*-topology. Using this and the fact that $\mathbf{C}^{\mathbf{l}}[a, b]$ is dense in $\mathbf{C}_{0}(a, b)$ one obtains the result.

Related work. Cf. [17] and [18] for related work by Ju. A. Brudnyi and J. Bergh and J. Peetre. Cf. also Bergh and Peetre's review of [17] (Zentralblatt, vol. 299, no. 41007). These authors refer to $\mathbf{N}^{p, n}$ and generalizations as spaces of functions of generalized bounded variation. There is some overlap between their results and those of the present paper and of [1], [2], some of which were announced already in 1973 [15] and 1974 [16]. However, many of our results here and in [2] appear to depend on the (smaller?) spaces $\mathbf{N}_{0}^{p, n}$, which Brudnyi and Peetre and Bergh do not apparently consider.

\section{REFERENCES}

1. H. G. Burchard, Splines (with optimal knots) are better, J. Appl. Anal. 3 (1974), 309-319.

2. H. G. Burchard and D. F. Hale, Piecewise polynomial approximation on optimal meshes, J. Approximation Theory 14 (1975), 128-147. MR 51 \# 10957.

3. P. L. Butzer and R. J. Nessel, Fourier analysis and approximation, Vol. 1, Academic Press, New York, 1971.

4. P. L. Butzer and K. Scherer, On the fundamental approximation theorems of D. Jackson, S. N. Bernstein and theorems of M. Zamansky and S. B. Steckin, Aequationes Math. 3 (1969), 170-185. MR 41 \#8897.

5. C. de Boor, On uniform approximation by splines, J. Approximation Theory 1 (1968), 219-235. MR 39 \# 1866.

6. Good approximation by splines with variable knots, Spline Functions and Approximation Theory (A. Meir and A. Sharma, editors), Birkhäüser, Basel, 1972, pp. 57-72.

7. D. S. Dodson, Optimal order approximation by polynomial spline functions, Thesis, Purdue Univ., 1972.

8. N. Dunford and J. Schwartz, Linear operators. Vol. I, Interscience, New York, 1958. MR 22 $\# 8302$.

9. G. Freud and V. A. Popov, Certain questions connected with approximation by splinefunctions and polynomials, Studia Sci. Math. Hungar. 5 (1970), 161-171. (Russian) MR 42 \#2225.

10. J. P. Kahane, Teoria constructiva de funciones, Cursos y Sem. Mat., Fasc. 5, Univ. Buenos Aires, Buenos Aires, 1961. MR 26 \#2728.

11. D. E. McClure, Nonlinear segmented function approximation and analysis of line patterns, Tech. Report, Div. Appl. Math., Brown Univ. 1973.

12. G. M. Phillips, Error estimates for best polynomial approximations, Approximation Theory (Proc. Sympos., Lancaster, 1969), A. Talbot, Editor, Academic Press, London, 1970, pp. 1-6. MR 43 \#3703.

13. H. B. Curry and I. J. Schoenberg, On Pólya frequency functions. IV: The fundamental spline functions and their limits, J. Analyse Math. 17 (1966), 71-107. MR 36 \#1884.

14. C. R. de Boor, Good approximation with variable knots. II, Conf. on the Numerical Solution of Differential Equations (Dundee, 1973), Lecture Notes in Math., vol. 363, Springer-Verlag, Berlin and New York, 1974, pp. 12-20.

15. H. G. Burchard and D. F. Hale, Direct and converse theorems for piecewise polynomial 
approximation on optimal partitions, Notices Amer. Math. Soc. 20 (1973), A-277. Abstract \#73T-B100.

16. H. G. Burchard, Degree of convergence of piecewise polynomial approximation on optimal meshes. II, Notices Amer. Math. Soc. 21 (1974), A-639. Abstract \# 719-B11.

17. Ju. A. Brudnyi, Spline approximation, and functions of bounded variation, Dokl. Akad. Nauk SSSR 215 (1974), 511-515 = Soviet Math. Dokl. 15 (1974), 518-521. MR 52 \#6249.

18. J. Bergh and J. Peetre, On the spaces $V_{p}(0<p<\infty)$, Tech. Report 1974:7, Dept. of Math., Univ. of Lund, 1974.

Department of Mathematics, Oklahoma State University, Stillwater, Okilahoma 74074 